Draft version August 8, 2018

Preprint typeset using LATEX style emulateapj v. 08/22/09

\title{
HIGH RESOLUTION IMAGING OF WARM AND DENSE MOLECULAR GAS IN THE NUCLEAR REGION OF THE LUMINOUS INFRARED GALAXY NGC 6240
}

\author{
Daisuke Iono $^{1}$, Christine D. Wilson ${ }^{2,3}$, Shigehisa Takakuwa ${ }^{1}$, Min S. Yun ${ }^{4}$, Glen R. Petitpas ${ }^{2}$, Alison B. Peck $^{2}$, \\ Paul T. P. Ho ${ }^{2,6}$, Satoki Matsushita ${ }^{6}$, Ylva M. Pinlstrom ${ }^{5}$, Zhong Wang ${ }^{2}$ \\ Draft version August 8, 2018
}

\begin{abstract}
We present $\sim 2^{\prime \prime}$ resolution $\mathrm{CO}(3-2), \mathrm{HCO}^{+}(4-3)$ and $880 \mu \mathrm{m}$ continuum images of the luminous infrared galaxy NGC 6240 obtained at the Submillimeter Array. We find that the spatially resolved $\mathrm{CO}(3-2), \mathrm{HCO}^{+}(4-3)$ and the $880 \mu \mathrm{m}$ emission peaks between the two nuclear components that are both known to harbor AGNs. Our Large Velocity Gradient (LVG) analysis performed on each velocity channel suggests that the peak of the molecular gas emission traced in our observations is warm $(\mathrm{T}=$ $20-100 \mathrm{~K})$, dense $\left(n_{\mathrm{H}_{2}}=10^{5.0-5.4} \mathrm{~cm}^{-3}\right)$ and moderately optically thin $(\tau=0.2-2)$ in the central $1 \mathrm{kpc}$. We also find large column densities of $\sim 10^{23} \mathrm{~cm}^{-2}$. Such extreme conditions are observed over $\sim 300 \mathrm{~km} \mathrm{~s}^{-1}$ centered around the $\mathrm{CO}$ derived systemic velocity. The derived molecular gas mass from the $\mathrm{CO}(3-2)$ emission and a $\mathrm{CO}$-to- $\mathrm{H}_{2}$ conversion factor commonly used for ULIRGs is $(6.9 \pm 1.7) \times 10^{9} \mathrm{M}_{\odot}$, and this is consistent with the mass derived from previous CO (2-1) observations. The gas is highly turbulent in the central kpc $\left(\Delta v_{\text {FWZI }} \sim 1175 \mathrm{~km} \mathrm{~s}^{-1}\right)$. Furthermore, possible inflow or outflow activity is suggested from the $\mathrm{CO}(3-2)$ velocity distribution. We tentatively state that $3.5 \times 10^{8} \mathrm{M}_{\odot}$ of isolated $\mathrm{CO}(3-2)$ emission seen west of the northern disk may be associated with outflows from starburst superwinds, but the gas outflow scenario from one of the central AGN is not completely ruled out. Piecing all of the information together, the central region of NGC 6240 harbors $2 \mathrm{AGNs}, \sim 10^{10} \mathrm{M}_{\odot}$ of molecular gas mass, $5 \times 10^{7} \mathrm{M}_{\odot}$ of dust mass, and has possible evidence of inflow and outflow activity.

Subject headings: galaxies: formation, galaxies: interaction, galaxies: starburst, galaxies: kinematics and dynamics, galaxies: individual (NGC 6240)
\end{abstract}

\section{INTRODUCTION}

(Ultra) Luminous Infrared Galaxies [(U)LIRGs] are systems discovered in the local universe that emit extremely large luminosity in the far infrared (see Sanders \& Mirabel 1996; Lonsdale, Farrah \& Smith 2006, for review). Their disturbed optical/NIR morphologies (e.g. Armus, Heckman \& Milev 1987; Surace et al. 1998; Farrah et al. 2001; Bushouse et al. 2002) and the highly compact molecular gas emission (Downes \& Solomon 1998) suggest that collisions/mergers of gas rich galaxies and subsequent radial inflow of gas toward the central region is primarily responsible for triggering extensive bursts of nuclear star formation (Scoville et al. 2000), feeding the obscured central AGN (Imanishi, Dudley \& Maloney 2006), or most likely, both (Genzel et al. 1998). Such inflow activity was already predicted in early numerical simulation studies that involve collisions of two massive gas rich galaxies (Barnes \& Hernquist 1996; Mihos \& Hernquist 1996; Iono, Yun \& Mihos 2004). More recent sim-

\footnotetext{
${ }^{1}$ National Astronomical Observatory of Japan, 2-21-1 Osawa, Mitaka, Tokyo 181-0015, Japan; d.iono@nao.ac.jp

${ }^{2}$ Harvard-Smithsonian Center for Astrophysics, 60 Garden Street, Cambridge, MA 02138

${ }^{3}$ Department of Physics and Astronomy, McMaster University, Hamilton, ON L8S 4M1, Canada

${ }^{4}$ Department of Astronomy, University of Massachusetts, Amherst, MA 01003

${ }^{5}$ Department of Physics and Astronomy, University of New Mexico, 800 Yale Boulevard NE, Albuquerque, NM 87131

6 Academia Sinica Institute of Astronomy and Astrophysics, P.O. Box 23-141, Taipei 106, Taiwan, R.O.C.
}

ulation studies that include a more realistic set of gas physics and the evolution of a central black hole have tied the merger event with radial gas inflow, central starbursts, black hole feeding, and a powerful gas outflow (Springel, Di Matteo \& Hernquist 2005; Hopkins et al. 2005; Di Matteo, Springel \& Hernquist 2005; Narayanan et al. 2006). These simulations have predicted a self-consistent evolutionary scenario from mergers to ULIRGs with a brief optical QSO phase (Hopkins et al. 2006).

High resolution observations of molecular gas tracers in (U)LIRGs and have been limited to low $J$ transition $\mathrm{CO}$ emission obtained at $\mathrm{mm}$ interferometers. Past single dish measurements of dense gas tracers such as HCN (Gao \& Solomon 2004) have found that molecular gas in (U)LIRGs is highly compressed and dense. Thus, obtaining sensitive and high spatial resolution images of high excitation molecular gas emission will provide extremely valuable information regarding the star formation process during a merger and will further constrain the physics implemented in the evolution simulations. In addition, recent surveys of molecular gas traced in high $J$ transition CO in high- $z$ galaxies (Greve et al. 2005; Tacconi et al. 2006) will allow us to conduct a direct comparison of the gas properties with local counterparts.

Here we present interferometric $\mathrm{CO}(3-2), \mathrm{HCO}^{+}(4-$ 3 ), and $880 \mu \mathrm{m}$ continuum observations of the luminous infrared galaxy (LIRG) NGC 6240 obtained at the SMA (Ho, Moran \& Lo 2004). Both molecular lines are known to trace warmer and denser molecular gas than the commonly observed mm lines such as CO $(1-0)$ or CO $(2-1)$. 
Past SMA observations toward infrared bright galaxies suggest that the distribution of the CO (3-2) emission is significantly different from the lower transition lines of CO (Iono et al. 2004; Wang et al. 2004).

\subsection{Multi-Wavelength Properties of NGC 6240}

NGC 6240 is an infrared luminous merger $\left(\mathrm{L}_{\mathrm{FIR}}=\right.$ $\left.3.5 \times 10^{11} \mathrm{~L}_{\odot}\right)($ Yun \& Carilli 2002) at a distance of $\mathrm{D}_{L}=107 \mathrm{Mpc}\left(1^{\prime \prime}=493 \mathrm{pc}\right)^{7}$. Its disturbed optical morphology and the compact double nuclei suggest that NGC 6240 is a late stage merger. The presence of AGN and extended starbursts were already suggested from early X-ray observations using a variety of spaceborne instruments (i.e. Ikebe et al. 2000, and references therein). More recently, Chandra has resolved the central region into two AGNs separated by $\sim 1^{\prime \prime}$ (Komossa et al. 2003), providing significant evidence that NGC 6240 is a merger of two AGN host galaxies. Recent XMMNewton spectroscopy has revealed a significant contribution of starburst activity to the extended X-ray emission (Boller et al. 2003; Netzer et al. 2005).

Optical and infrared observations using ground based and spaceborne telescopes have also been used to study this galaxy intensively. $H S T$ and Keck imaging have resolved each nucleus into several distinct components (Gerssen et al. 2004; Max et al. 2005) with an apparently dusty central region. A large velocity gradient in ionized gas has been identified using optical spectroscopy (Gerssen et al. 2004). Near IR imaging has also shown the presence of two $K$-band nuclei (Scoville et al. 2000; Bogdanovic et al. 2003). Signatures of starbursts appear to dominate the MIR spectrum (Lutz et al. 2003). Mid IR spectroscopy using IRS on the Spitzer Space Telescope suggest a minor $(\sim 3-20 \%)$ contribution of the AGN to the bolometric luminosity (Armus et al. 2006), but mid infrared spectra obtained at Keck suggest that the dominant power-source could be explained by starbursts or an AGN (Egami et al. 2006). Optical spectroscopy using Subaru has revealed significant $\mathrm{H}_{2}$ emission 0 "' 2 north of the southern nucleus, suggesting that the medium between the two nuclei is dominated by starburst superwinds from the southern nucleus (Ohyama et al. 2000). Finally, a star formation rate of $61 \pm 30 \mathrm{M}_{\odot} \mathrm{yr}^{-1}$ is obtained from a fit to the FIR SED (Yun \& Carilli 2002) and using the Schmidt law derived in Kennicutt (1998) to covert to the Star Formation Rate (SFR).

Interferometric millimeter studies have identified significant amounts of molecular gas in NGC 6240 (Brvant \& Scoville 1999; Tacconi et al. 1999; Nakanishi et al. (2005). The bulk of cold and dense gas peaks between the two optical/X-ray nuclei, close to the region where significant $\mathrm{H}_{2}$ emission (Ohyama et al. 2000) is found (Figure 1). Millimeter continuum emission also peaks in the same region. Molecular gas kinematics traced in $\mathrm{CO}(2-1)$ emission show a large velocity gradient between the two nuclei, suggesting that $\sim 10^{9} \mathrm{M}_{\odot}$ of molecular gas is in the process of accumulating in the new dynamical center between the two nuclei.

MERLIN observations (Beswick et al. 2001) have detected both AGNs with highly disturbed $\mathrm{H}$ I gas in absorption. Further, high resolution VLBA observations

\footnotetext{
7 Adopting $\mathrm{H}_{0}=70 \mathrm{~km} \mathrm{~s}^{-1} \mathrm{Mpc}^{-1}, \Omega_{M}=0.3, \Omega_{\Lambda}=0.7$
}

(Gallimore \& Beswick 2004) have detected two compact radio sources (N1 and S1 in Figure 1) and an additional component (S2) 0.'2 north of the southern nucleus (S1). Gallimore \& Beswick (2004) attribute N1 and S1 to compact radio emission associated with the AGNs, and $\mathrm{S} 2$ as a radio supernova or material ejected from $\mathrm{S} 1$. An $\mathrm{H}_{2} \mathrm{O}$ maser detected near S1 (Hagiwara, Diamond \& Miyoshi 2003) is attributable to either a jet associated with the southern AGN, or with shocks caused by the immense collision. From their low resolution VLA maps, Colbert et al. (1994) have found significant centimeter emission west of N1. This emission is resolved into four distinct components, and they suggest shell material from galactic super-wind as an origin of the emission.

This article is organized as follows. In $\S 2$, we present our observational method and the detailed calibration scheme adopted for the SMA observations. The CO (32 ) and $\mathrm{HCO}^{+}(4-3)$ moment maps are presented in $\S 3$, along with the $880 \mu \mathrm{m}$ continuum image which was made by averaging the emission in the line free channels. $\S 4$ focuses on the physical properties of gas. We provide a discussion of our results in $\S 5$, and conclude this paper in $\S 6$.

\section{OBSERVATIONS AND DATA REDUCTION}

NGC 6240 was observed on October 9 and 14, 2005 using both the compact (unprojected baselines $=16-69$ meters) and extended configurations (unprojected baselines $=50-182$ meters) of the SMA. These data were taken in excellent atmospheric conditions $\left(\tau_{230}=0.05-\right.$ 0.06). The digital correlator was configured with 2048 channels for the entire $2 \mathrm{GHz}$ bandwidth for each sideband. The receivers were tuned to the redshifted $\mathrm{CO}(3-$ 2) $\left(\nu_{\text {rest }}=345.796 \mathrm{GHz}\right)$ emission line in the lower sideband (LSB), which allows us to simultaneously obtain the $\mathrm{HCO}^{+}(4-3)\left(\nu_{\text {rest }}=356.734 \mathrm{GHz}\right)$ line in the upper sideband (USB). The adopted tuning frequency was $337.533 \mathrm{GHz}$ (LSB) using $v_{\text {sys }}=7339 \mathrm{~km} \mathrm{~s}^{-1}$. We used $\alpha(\mathrm{J} 2000)=16^{\mathrm{h}} 52^{\mathrm{m}} 58.9^{\mathrm{s}}$ and $\delta(\mathrm{J} 2000)=2^{\circ} 24^{\prime} 04.00^{\prime \prime}$ (Laurent-Muehleisen et al. 1997) as our phase reference center. Initial data calibration were carried out in the IDL based SMA calibration package MIR, where 3C279, 3C454.3 and Uranus were used for bandpass calibration. Absolute flux calibration was performed using Uranus, and time dependent gain calibration was performed using the 1.6 Jy QSO 1743-038 (14 degrees away from NGC 6240) and the 1.4 Jy QSO 1549+026 (16 degrees away from NGC 6240) as references.

Synthesis imaging was performed in MIRIAD. Channel maps with $25 \mathrm{~km} \mathrm{~s}^{-1}$ velocity resolution were made for the CO (3-2) line. We further varied the visibility weighting from natural (i.e. maximum sensitivity) which gave a synthesized beam size of 2 .' $0 \times 1$ ". 8 (position angle $=49$ degrees; Figure 2 (left)) to uniform (i.e. maximum angular resolution) which gave a synthesized beam size of 1 ). $1 \times 00^{\prime \prime} .8$ (position angle $=49$ degrees; Figure 2 (right)). Unity weights are assigned for each visibility point in natural weighting, giving the best $\mathrm{S} / \mathrm{N}$ and the coarsest angular resolution. On the other hand, uniform weighting gives the highest angular resolution at the cost of lower $\mathrm{S} / \mathrm{N}$. This is achieved by weighting each visibility point by the reciprocal of the number of data points found in a grid centered around each visibility point. The rms noise level was $22 \mathrm{mJy}$ and $78 \mathrm{mJy}$ for 
the natural and uniformly weighted maps respectively. In addition, in order to derive the peak coordinates of the CO (3-2) emission, we have constructed an image using only the longest baseline data which gave a synthesized beam size of $0 . ! 9 \times 0.5$ (position angle $=41 \mathrm{de}-$ grees). For the natural weighted beam, the first sidelobes are significant at the $50 \%$ level $\sim 11^{\prime \prime}$ north and south of the main synthesized beam. Sidelobes at $10 \%$ level are seen in various positions, the effect of which should be negligible with proper deconvolution. For the uniformly weighted beam, the first significant $(\sim 30 \%)$ sidelobes are located $\sim 5^{\prime \prime}$ north-south of the main beam. CLEANing was performed down to 1.5 times the rms noise level of each channel map. For the deconvolution algorithm, we chose CLEAN instead of the Maximum Entropy Method (MEM) since the emission is strong and relatively compact with both the natural and uniformly weighted beams.

The $\mathrm{HCO}^{+}(4-3)$ channel maps were made using natural weighting, which gave a $2^{\prime \prime} .0 \times 11^{\prime \prime} 7$ (position angle $=42$ degrees) beam. We note that the $\mathrm{HCO}^{+}(4-3)$ emission occurs near the edge of the bandpass, and these observations may be missing flux at the low velocity end. Continuum subtraction was performed by removing a linear baseline in the visibilities in both sidebands. The $880 \mu \mathrm{m}$ continuum image was made by adding all of the line free channels in the bandpass of both sidebands. The rms noise level for the continuum map was $6 \mathrm{mJy}$. From the $\mathrm{S} / \mathrm{N}$ of the maps, we estimate the astrometric accuracy of the $\mathrm{CO}(3-2)$ maps presented here to be $<0$ " 1 , and $\sim 0^{\prime \prime} 1$ for the $\mathrm{HCO}^{+}(4-3)$ and $880 \mu \mathrm{m}$ maps.

\section{RESULTS}

The channel maps of CO (3-2) emission are provided in Figure 3. Figure 4 (left) shows the integrated intensity map of the natural weighted $\mathrm{CO}(3-2)$ emission, and Figure 4 (right) shows the uniformly weighted image of the central $6^{\prime \prime}$. Figure 5 show the velocity distribution with the same angular resolution as in Figure 4. The velocity distribution of the western complex (WC hereafter; see below) alone is shown in Figure 6. The integrated intensity maps are clipped at 1.5 times the rms in each channel, whereas the velocity distribution maps are clipped at 3 times the rms. The position velocity maps obtained along the two AGNs are shown in Figure 7 . Figure 8 shows the $\mathrm{HCO}^{+}(4-3)$ and $880 \mu \mathrm{m}$ emission images of NGC 6240 . A summary of the derived parameters is shown in Table 1.

\section{1. $C O$ (3-2) Emission}

\subsubsection{Distribution}

The size of the $\mathrm{CO}(3-2)$ emission region in the integrated intensity map of Figure 4 (left) extends $4.4 \mathrm{kpc}$ in both north-south and east-west directions. The coordinates of the peak determined from the highest resolution map obtained by just using data in the extended configuration are $\alpha(\mathrm{J} 2000)=16^{\mathrm{h}} 52^{\mathrm{m}} 58.9^{\mathrm{s}}$ and $\delta(\mathrm{J} 2000)=$ $2^{\circ} 24^{\prime} 03^{\prime \prime} .70$. This is coincident with the radio component $(\mathrm{S} 2)$ at $\alpha(\mathrm{J} 2000)=16^{\mathrm{h}} 52^{\mathrm{m}} 58.8994^{\mathrm{s}}$ and $\delta(\mathrm{J} 2000)=$ $2^{\circ} 24^{\prime} 03^{\prime \prime} .5925$ (error $=0.004^{\prime \prime}$ ) (Gallimore \& Beswick 2004) to within 0 .' 1 which is comparable to the astrometric accuracy of the SMA. While the inner region of the map shown in Figure 4 (left) is not spatially resolved with this map, there are several distinct $\sim 1 \mathrm{kpc}$ scale features that dominate the emission in the periphery; (1) the emission that extends to the east, (2) the emission that extends to the north, and (3) the isolated emission west of the main $\mathrm{CO}(3-2)$ concentration (WC).

The $\sim 2 \mathrm{kpc}$ eastern extension is significant in the velocity range of 6964 to $7489 \mathrm{~km} \mathrm{~s}^{-1}$ (see Figure 3), and appears to originate from the southeastern edge of the central emission region. On the other hand, the $\sim 2 \mathrm{kpc}$ northern extension is significant in the velocity range 7264 to $7564 \mathrm{~km} \mathrm{~s}^{-1}$, and appears to display a slight curve toward the east. The isolated component (WC) was detected $\sim 9^{\prime \prime}(4.4 \mathrm{kpc})$ west of the main CO $(3-2)$ peak, and this emission is significant in the velocity range 7114 to $7714 \mathrm{~km} \mathrm{~s}^{-1}$. No significant optical counterpart is associated with WC. In addition, none of the past interferometric $\mathrm{CO}$ observations have identified a similar feature (Brvant \& Scoville 1999; Tacconi et al. 1999; Nakanishi et al. 2005), but radio continuum observations have detected significant extension of synchrotron emission in the same region (Colbert et al. 1994).

The total integrated intensity from the full resolution map including WC is $2834 \pm 20 \mathrm{Jy} \mathrm{km} \mathrm{s}^{-1}$, recovering $90 \%$ of the total flux measured using a single dish telescope $\left(3180 \pm 460 \mathrm{Jy} \mathrm{km} \mathrm{s}{ }^{-1}\right.$; Greve et al. 2006). Using the $\mathrm{CO}-\mathrm{H}_{2}$ conversion factor appropriate for ULIRGs (i.e. $X=0.8 \pm 0.2 \mathrm{M}_{\odot}\left(\mathrm{K} \mathrm{km} \mathrm{s}^{-1}\right)^{-1} \mathrm{pc}^{2} ;$ Downes \& Solomon 1998), and assuming $\mathrm{L}_{\mathrm{CO}(3-2)}^{\prime}=\mathrm{L}_{\mathrm{CO}(1-0)}^{\prime}, \mathrm{L}_{\mathrm{CO}(3-2)}^{\prime}=$ $(8.6 \pm 0.1) \times 10^{9} \mathrm{~K} \mathrm{~km} \mathrm{~s}^{-1} \mathrm{pc}^{2}$ translates to $\mathrm{M}_{\mathrm{H}_{2}}=$ $(6.9 \pm 1.7) \times 10^{9} \mathrm{M}_{\odot}$. The peak of the CO (3-2) emission gives a peak $\mathrm{H}_{2}$ column density of $\mathrm{N}_{\mathrm{H}_{2}}=(2.0 \pm$ $0.3) \times 10^{23} \mathrm{~cm}^{-2}$ using $X=0.8 \mathrm{M}_{\odot}\left(\mathrm{K} \mathrm{km} \mathrm{s}^{-1}\right)^{-1} \mathrm{pc}^{2}$. The peak brightness temperature observed with the $\sim 2^{\prime \prime}$ beam is $8.6 \mathrm{~K}$. The integrated intensity of WC alone is $(143 \pm 20) \mathrm{Jy} \mathrm{km} \mathrm{s}{ }^{-1}$, which translates to $\mathrm{M}_{\mathrm{H}_{2}}=$ $(3.5 \pm 1.0) \times 10^{8} \mathrm{M}_{\odot}$.

The higher resolution image afforded by adopting uniform weighting shown in Figure 4 (right) resolves out the extended structure, revealing the compact CO (32) emission that dominates the central $1.5 \mathrm{kpc}$. The integrated intensity derived from this map is $1839 \pm$ $20 \mathrm{Jy} \mathrm{km} \mathrm{s}^{-1}$ and this is $65 \%$ of the total intensity derived from the full resolution image. The integrated intensity translates to $\mathrm{M}_{\mathrm{H}_{2}}=(4.5 \pm 1.1) \times 10^{9} \mathrm{M}_{\odot}$ assuming $X=0.8 \pm 0.2 \mathrm{M}_{\odot}\left(\mathrm{K} \mathrm{km} \mathrm{s}^{-1}\right)^{-1} \mathrm{pc}^{2}$. The CO (3-2) emission arises primarily in a compact structure centered between the two nuclei and slightly extended in the direction defined by the line connecting the two.

\subsubsection{Kinematics}

The total CO (3-2) FWZI linewidth of $1175 \mathrm{~km} \mathrm{~s}^{-1}$ is extremely large (see Figure 31). The large linewidth, particularly in the medium surrounding the two AGNs, suggests extremely turbulent gas. While it is possible that the bulk of the gas seen in Figure 5 (left) is dominated by turbulent gas stirred during the massive collision, the northeast-southwest velocity gradient centered around the northern nucleus (N1) may be suggestive of a disk rotation. The higher resolution image in Figure 5 (right) also shows this compact, rotation feature. Rapid changes in velocity of $\Delta v \sim 100 \mathrm{~km} \mathrm{~s}^{-1}$ are seen along the northern extended feature that appears to connect to this rotating disk, possibly suggesting collimated 
gas streaming toward the direction close to the line of sight. A steep velocity gradient like this is suggestive of gas inflow or outflow. The velocity gradient in the vicinity of the southern nucleus (S1) is less obvious, possibly due to the dominance of local non-circular motion, or a face-on orientation of a rotating disk. Although our current data does not have high enough angular resolution to distinguish the exact origin, if the southern nucleus has a face-on rotating disk, then the large shift in velocity of $\sim 250 \mathrm{~km} \mathrm{~s}^{-1}$ that begins from the south of S1 and along the eastern extension may also suggest inflow or outflow activity. The velocity distribution (Figure 5 (right)) shows that the systemic velocities of the two nuclei are offset by $\sim 100 \mathrm{~km} \mathrm{~s}^{-1}$. The emission in WC is significant in the velocity range 7114 to $7564 \mathrm{~km} \mathrm{~s}^{-1}$ (see Figure 3), and does not show any significant evidence of a rotation in the moment map (Figure 6). An overall velocity shift from the northern part to the southern part of $\mathrm{WC}$ is evident.

The position-velocity diagrams (PVD) sliced along the two AGNs (N1 and S1) and centered exactly halfway between the two AGNs are shown in Figure 7 for different resolution maps. Figure [7 (left) clearly shows that the linewidth near the two nuclei is extremely large, with emission slightly lopsided toward the redshifted velocities $\left(v>0 \mathrm{~km} \mathrm{~s}^{-1}\right)$. The PVD obtained with the uniformly weighted map resolves out the extended emission in the velocity extrema, showing more details in the central $\pm 200 \mathrm{~km} \mathrm{~s}^{-1}$ (Figure 7 (middle)). Similar to Figure 7 (left), the peak of the emission is near the systemic velocity of $7339 \mathrm{~km} \mathrm{~s}^{-1}$. In contrast, the PVD obtained (Figure 7 (right)) with the highest angular resolution map completely resolves the central emission into two distinct peaks separated by $\sim 70 \mathrm{~km} \mathrm{~s}^{-1}$ and $\sim 0$ ! 2 (100 pc). This double peaked morphology in the PVD suggests overlapping molecular cloud complexes in the medium between the two AGNs. The fact that these two peaks are nearly spatially coexistent, but separated in velocity further strengthens the argument that the gas kinematics in the central region is highly turbulent and non-circular.

\section{2. $\mathrm{HCO}^{+}(4-3)$ Emission}

The distribution of the $\mathrm{HCO}^{+}(4-3)$ emission (Figure $8($ left $)$ ) is highly compact, and it is concentrated in the central region with a northeast-southwest elongation of $4^{\prime \prime} \times 2^{\prime \prime}(2 \times 1 \mathrm{kpc})$, with an additional slight extension toward the southeast. The peak of the $\mathrm{HCO}^{+}(4-$ $3)$ emission is $\alpha(\mathrm{J} 2000)=16^{\mathrm{h}} 52^{\mathrm{m}} 58.9^{\mathrm{s}}$ and $\delta(\mathrm{J} 2000)=$ $2^{\circ} 24^{\prime} 03^{\prime \prime} 8$, and this is consistent with the peak of the CO (3-2) to within the astrometric accuracy of the SMA. The integrated intensity is $(28 \pm 4) \mathrm{Jy} \mathrm{km} \mathrm{s}^{-1}$, recovering $40 \%$ of the total flux measured using a single dish telescope $\left(78 \pm 16 \mathrm{Jy} \mathrm{km} \mathrm{s}^{-1}\right.$; Greve et al. 2006). The peak brightness temperature observed with the $\sim 2^{\prime \prime}$ beam is $0.5 \mathrm{~K}$. The highly compact and low $\mathrm{S} / \mathrm{N}$ emission prevents us from constructing an interpretable velocity distribution map. The $\mathrm{HCO}^{+}(1-0)$ emission obtained with a coarser synthesized beam also peaks at a similar location, although the emission is more extended in the northwest-southeast direction (Nakanishi et al. 2005).

\section{3. $880 \mu m$ Continuum Emission}

Similar to the $\mathrm{CO}(3-2)$ and $\mathrm{HCO}^{+}(4-3)$ emission, the $880 \mu \mathrm{m}$ emission also peaks between the two nuclei, near the radio continuum feature $\mathrm{S} 2$. The $880 \mu \mathrm{m}$ peak is also consistent with the $1.3 \mathrm{~mm}$ peak within the uncertainties of both measurements (Figure 1). The coordinates of the $880 \mu \mathrm{m}$ peak are $\alpha(\mathrm{J} 2000)=16^{\mathrm{h}} 52^{\mathrm{m}} 58.9^{\mathrm{s}}$ and $\delta(\mathrm{J} 2000)=2^{\circ} 24^{\prime} 03^{\prime \prime} .4$. The extension to the east is coincident with the similar extension seen in the CO (32) map, but the continuum is much shorter. The size of the $880 \mu \mathrm{m}$ emitting region is $6^{\prime \prime} 0 \times 3^{\prime \prime} .5(2.9 \times 1.7 \mathrm{kpc})$. The total flux detected from the SMA observations is $(105 \pm 6) \mathrm{mJy}$, and this is consistent with the true single dish continuum measurement of $96 \mathrm{mJy}$, which is calculated from the SCUBA $850 \mu \mathrm{m}$ flux (Klaas et al. 2001) and subtracting the contribution of the CO (3-2) emission using the formulae given in Seaquist et al. (2004). Assuming that all of the $880 \mu \mathrm{m}$ emission is associated with dust, the derived dust mass in the central $2 \mathrm{kpc}$ is $(4.7 \pm 1.4) \times 10^{7} \mathrm{M}_{\odot}$ using the formula described in Wilson et al. (2006 in prep). This yields a gas-to-dust mass ratio of $(147 \pm 57)$ in the central $2 \mathrm{kpc}$ region (see $\S 3.1$ for the gas mass). Although uncertainties in both mass calculations are large, this is in agreement with the values typically observed in the Galaxy. The peak of the continuum suggests that the bulk of the dusty starburst activity occurs in this region. This is in contrast to previous optical/NIR imaging studies (e.g. Pasquali. Gallagher \& de Grijs 2004) where the visible starburst activity is concentrated near the two optical/Xray nuclei, suggesting that the SFRs $\left(2.8-412 \mathrm{M}_{\odot} \mathrm{yr}^{-1}\right.$; Pasquali, Gallagher \& de Grijs 2004) derived using these measurements are likely underestimated.

\section{ANALYSIS OF THE PHYSICAL PROPERTIES}

\subsection{Large Velocity Gradient Modeling}

Our new $\mathrm{CO}(3-2)$ and $\mathrm{HCO}^{+}(4-3)$ data allow us to investigate the physical conditions of the gas by taking the line ratios with previously published CO (1-0) and $\mathrm{HCO}^{+}(1-0)$ data (Nakanishi et al. 2005). Past multiline studies toward galaxies have been limited to line ratios obtained from single dish data (e.g. Aalto et al. 1995; Petitpas \& Wilson 2000; Yao et al. 2003) or the ratios between the integrated intensities for nearby systems (e.g. Matsushita et al. 1999; Mao et al. 2000). Here we present line ratios obtained in each velocity channel, and investigate the gas properties by comparing the ratios with the theoretical predictions obtained from Large Velocity Gradient (LVG) models (Goldreich \& Kwan 1974; Takakuwa, Mikami \& Saito 1998). We assume here that both $\mathrm{CO}$ and $\mathrm{HCO}^{+}$emission in the central $\mathrm{kpc}$ arise from the same molecular cloud. Although it is suggested that the filling factors of denser gas tracers may be smaller than the commonly observed CO (1-0) line (e.g. Aalto et al. 1995), here we assume for simplicity that the (beam and volume) filling factors between $\mathrm{CO}(3-2)$ and $\mathrm{CO}(1-0)$, as well as $\mathrm{HCO}^{+}(4-3)$ and $\mathrm{HCO}^{+}(1-0)$ are similar. We note that, although the $\mathrm{HCO}^{+}(4-3)$ emission recovers only $40 \%$ of the total single dish flux (§3.2), the main focus of this analysis is to investigate the gas properties in the central $\sim 1^{\prime \prime}$ where the missing flux should be negligible. By comparing the line ratios between $\mathrm{CO}(3-2)$ and $\mathrm{CO}(1-0)$, and also $\mathrm{HCO}^{+}(4-3)$ and $\mathrm{HCO}^{+}(1-0)$, these assumptions allow a direct compari- 
son with LVG models that does not depend on the filling factor.

All data were convolved to the angular resolution of the CO $(1-0)$ image $(2$.' $2 \times 2$ '. 1 ; lowest angular resolution among the four data cubes), regridded to the same velocity resolution of $56 \mathrm{~km} \mathrm{~s}^{-1}$, and two line ratio cubes, $\mathrm{CO}(3-2) / \mathrm{CO}(1-0)\left(R_{\mathrm{CO}}\right)$ and $\mathrm{HCO}^{+}(4-3) / \mathrm{HCO}^{+}(1-$ $0)\left(R_{\mathrm{HCO}^{+}}\right)$were formed. The pixel-to-pixel correlation between the two ratio cubes were obtained and are shown as crosses in Figure 9. In order to show the regions with significant detection in all four transitions, only the data above two sigma in each channel are plotted. In brightness temperature, the two sigma cutoffs are $0.20 \mathrm{~K}, 0.80 \mathrm{~K}, 0.14 \mathrm{~K}$, and $0.26 \mathrm{~K}$, for CO (3-2), CO (1$0), \mathrm{HCO}^{+}(4-3)$, and $\mathrm{HCO}^{+}(1-0)$ respectively. Since the $\mathrm{HCO}^{+}(4-3)$ emission was only detected in the central 100 to $200 \mathrm{~km} \mathrm{~s}^{-1}$, only the densest gas in the central $<2^{\prime \prime}(1 \mathrm{kpc})$ is traced using this method.

In order to understand the correlations seen in Figure 9 better, a series of LVG calculations were performed. We adopt an LVG analysis that assumes a single cloud with spherical geometry, which requires three independent parameters as inputs: the $\mathrm{H}_{2}$ density $\left(n_{\mathrm{H}_{2}}\right)$, kinetic temperature $\left(\mathrm{T}_{\mathrm{K}}\right)$, and the molecular abundance per unit velocity gradient $(X /(d v / d r))$. The collision rates were obtained from Flower \& Launay (1985) for $\mathrm{CO}$ and Flower (1999) for $\mathrm{HCO}^{+}$. The quantity $X /(d v / d r)$, which is different for different species, is physically the most ambiguous parameter. In order to decrease the number of unknown parameters, we adopt a constraint to the relative abundance between $\mathrm{CO}$ and $\mathrm{HCO}^{+}\left(X_{\mathrm{HCO}^{+}} \sim 10^{-4} X_{C O}\right.$; Bergin \& Langer 1997) obtained from chemical evolution models. By varying the $\mathrm{CO}$ abundance per unit velocity gradient from $X_{C O} /(d v / d r)=10^{-4}$ to $10^{-9}\left(\mathrm{~km} \mathrm{~s}^{-1} \mathrm{pc}^{-1}\right)^{-1}$, $X_{C O} /(d v / d r) \sim 10^{-7}\left(\mathrm{~km} \mathrm{~s}^{-1} \mathrm{pc}^{-1}\right)^{-1}$ provides the most sensible explanation of the data. Changing $X_{C O} /(d v / d r)$ to $10^{-6}\left(\mathrm{~km} \mathrm{~s}^{-1} \mathrm{pc}^{-1}\right)^{-1}$ will shift the temperature (vertical) contours leftward (see Figure 10), yielding most of the molecular gas with temperatures in the range $100-200 \mathrm{~K}$. This is higher than the cold and warm dust temperatures predicted from a three component model by Armus et al. (2006) where they find cold $(27 \mathrm{~K})$, warm $(81 \mathrm{~K})$ and hot $(680 \mathrm{~K})$ dust components to coexist over the entire NGC 6240 system. Using $X_{C O} /(d v / d r)=10^{-8}\left(\mathrm{~km} \mathrm{~s}^{-1} \mathrm{pc}^{-1}\right)^{-1}$ will shift the temperature contours rightward, yielding most of the data points in $\mathrm{T}<40 \mathrm{~K}$. This is lower than the values predicted in the nearby starburst NGC 253 (Jackson et al. 1995), but consistent with the cold component dust in NGC 6240 suggested by Armus et al. (2006), assuming that the gas and dust are in thermal equilibrium.

Assuming that the gas temperature of $40-100 \mathrm{~K}$ is a reasonable representation of the molecular clouds in NGC 6240, then $X_{C O} /(d v / d r) \sim 10^{-7}\left(\mathrm{~km} \mathrm{~s}^{-1} \mathrm{pc}^{-1}\right)^{-1}$ provides the most sensible abundance that explains the data in all channels with significant emission (Figure 9). A tight constraint to the $\mathrm{H}_{2}$ density of $n_{\mathrm{H}_{2}}=$ $10^{5.0-5.4} \mathrm{~cm}^{-3}$ is obtained. The derived opacities range from $\tau=0.01-0.2$ for the $\mathrm{CO}(1-0)$ line, $\tau=0.2$ -2 for the $\mathrm{CO}(3-2)$ line, and $\tau=0.5-1$ for the $\mathrm{HCO}^{+}(4-3)$ line. The model predicts that the relative population of the $\mathrm{HCO}^{+}(1-0)$ line be inverted, thus giving negative opacities and excitation temperatures. The excitation temperatures $\left(\mathrm{T}_{e x}\right)$ derived from these analyses show that both CO lines are nearly thermalized (i.e. $\left.\mathrm{T}_{\mathrm{ex}, \mathrm{CO}(3-2)} \sim \mathrm{T}_{\mathrm{ex}, \mathrm{CO}(1-0)}\right)$.

Once these physical quantities are derived, it is now possible to constrain the beam filling factor of the $\mathrm{CO}$ emitting molecular gas. The observed line intensities are diluted by the $\sim 2^{\prime \prime}(1 \mathrm{kpc})$ beam, and thus the true flux is obtained by scaling the observed flux by the reciprocal of the filling factor. Figure 11 and 12 show the correlation between $\mathrm{T}_{\mathrm{CO}(3-2)}$ and $\mathrm{T}_{\mathrm{CO}(1-0)}$ in each $21 \mathrm{~km} \mathrm{~s}^{-1}$ channel where the emission of each line is significant (i.e. the central -133 to $187 \mathrm{~km} \mathrm{~s}^{-1}$ ). The filling factors of the $\mathrm{CO}$ lines, which are assumed to be the same between $\mathrm{CO}(3-2)$ and $\mathrm{CO}(1-0)$ as well as in all velocity channels, are varied from 1 (no scaling; Figure 11) to 0.2 (multiply the observed brightness temperatures by 5; Figure 12). The lower limit to the filling factor of 0.2 is obtained by adjusting the filling factor until the highest observed points reach $n_{\mathrm{H}_{2}} \lesssim 10^{5.4} \mathrm{~cm}^{-3}$, a constraint given by the $\mathrm{R}_{\mathrm{CO}}-\mathrm{R}_{\mathrm{HCO}}+$ plot in Figure 9. A CO filling factor of unity gives the bulk of the gas in the temperature range of $40-200 \mathrm{~K}$ and density of $n_{\mathrm{H}_{2}} \sim 10^{4.0} \mathrm{~cm}^{-3}$. In this case, while the range of temperature is consistent with our earlier analysis (see above), the $\mathrm{H}_{2}$ density is an order of magnitude lower. A CO filling factor of 0.2 also yields a temperature range of $20-100 \mathrm{~K}$, and the range of $\mathrm{H}_{2}$ density is also comparable to our earlier analysis. These results suggest that the true CO filling factor is between 0.2 to 1 , but probably closer to 0.2 . A low value of the filling factor would be inconsistent with the previous studies by Downes \& Solomon (1998) where they suggest that the diffuse inter-clump medium may dominate the $\mathrm{CO}$ emission in ULIRGs, yielding a filling factor of unity.

An estimate of the $\mathrm{CO}$ beam filling factor allows us to derive the volume filling factor of the $\mathrm{CO}$ emitting gas (see Equation 3 of Sakamoto et al. 1994). Assuming that the CO (3-2) emission arises from GMCs of order $50 \mathrm{pc}$ each, and that the CO (3-2) emission region along the line of sight extends $4 \mathrm{kpc}$, the range of possible volume filling factor is $0.004-0.05$ for filling factors $0.2-1$. Changing the size of the GMC to $25 \mathrm{pc}(10 \mathrm{pc})$ gives 0.002 - $0.03(0.001-0.01)$ for the volume filling factor. Using the average density estimate from our LVG analysis (i.e. $n_{\mathrm{H}_{2}} \sim 10^{5.0} \mathrm{~cm}^{-3}$ ), the derived molecular gas mass is then $10^{9-10} \mathrm{M}_{\odot}$ in the central $0.5 \mathrm{kpc}(\mathrm{r}=0.25 \mathrm{kpc}) \mathrm{re}-$ gion. Our mass estimate is consistent with $6.9 \times 10^{9} \mathrm{M}_{\odot}$ derived using $\mathrm{X}=0.8 \mathrm{M}_{\odot}\left(\mathrm{K} \mathrm{km} \mathrm{s}^{-1}\right)^{-1} \mathrm{pc}^{2}$ (see $\S 3.1$ ), and with $(1-4) \times 10^{9} \mathrm{M}_{\odot}$ derived in the central $0.5 \mathrm{kpc}$ using CO (2-1) line emission (Tacconi et al. 1999). Assuming that the average density in the larger spatial extent is also $n_{\mathrm{H}_{2}}=10^{5.0} \mathrm{~cm}^{-3}$ gives $\sim 10^{10} \mathrm{M}_{\odot}$ in the central $1 \mathrm{kpc}(\mathrm{r}=0.5 \mathrm{kpc})$, and $\sim 10^{11} \mathrm{M}_{\odot}$ in the central $2 \mathrm{kpc}(\mathrm{r}=1 \mathrm{kpc})$ region. The mass estimate given in a larger region (i.e. $2 \mathrm{kpc}$ ) is probably an overestimate since the molecular gas density in the periphery of the nucleus is likely lower than the central $0.5 \mathrm{kpc}$. Since the density derived from the LVG analysis only accounts for the densest gas in the central region, a proper mass estimate must consider the density variation of the cloud in the entire $1175 \mathrm{~km} \mathrm{~s}^{-1}$ velocity width, which is not 
possible here because of the varying mass sensitivities among the four data cubes.

The LVG calculation also provides an estimate of the $\mathrm{H}_{2}$ column density from the relation $3.0 \times$ $10^{18}\left(\mathrm{X}_{\mathrm{CO}} /(\mathrm{dv} / \mathrm{dr})\right) n_{\mathrm{H}_{2}}=\mathrm{N}_{\mathrm{CO}} / \mathrm{dv}$. By adopting $X_{C O} /(d v / d r)=10^{-7}\left(\mathrm{~km} \mathrm{~s}^{-1} \mathrm{pc}^{-1}\right)^{-1}$ and $n_{\mathrm{H}_{2}}=10^{5.0} \mathrm{~cm}^{-3}$, we find $\mathrm{N}_{\mathrm{CO}} / \mathrm{dv}=3.0 \times$ $10^{16} \mathrm{~cm}^{-2}\left[\mathrm{~km} \mathrm{~s}^{-1}\right]^{-1}$. Using the standard abundance observed in galactic sources $\left(\mathrm{X}_{\mathrm{CO}} / \mathrm{X}_{\mathrm{H}_{2}}=10^{-4}\right.$; Blake et al. 1987) and $\mathrm{dv}=\mathrm{FWZI}=1175 \mathrm{~km} \mathrm{~s}^{-1}$, we obtain $\mathrm{N}_{\mathrm{H}_{2}}=$ $4.0 \times 10^{23} \mathrm{~cm}^{-2}$. This is in excellent agreement with our previous column density estimate derived using the peak CO (3-2) flux.

The peak brightness temperatures obtained in the $\mathrm{CO}(3-2)$ and $880 \mu \mathrm{m}$ observations (see $\S 3.1$ and $\S 3.3$ ) allow a confirmation of the properties derived above. In general, the brightness temperature and the true excitation temperature are related by $\mathrm{T}_{B}=f \mathrm{~T}_{e x}\left(1-e^{-\tau}\right)$, where $\mathrm{T}_{B}, f, \mathrm{~T}_{e x}$ and $\tau$ are the observed brightness temperature, beam filling factor, excitation temperature, and opacity respectively. Assuming the observed molecular clouds and the $\mathrm{CO}$ excitation are in thermal equilibrium coupled with dust (i.e. $\mathrm{T}_{e x} \sim \mathrm{T}_{K} \sim \mathrm{T}_{\text {dust }} \sim 49 \mathrm{~K}$ ), and using the range of filling factors of $f=0.2-1$ (see above), we obtain $\tau=0.2-1.2$, which is consistent with the opacities derived above. This is further evidence that the observed molecular clouds are moderately optically thin, and that they are close to thermal equilibrium. A similar analysis is performed on the dust continuum, and we derive the dust opacity $\tau_{d u s t}=0.003-0.01$ using the same filling factors as CO, suggesting that the cold dust traced in $880 \mu \mathrm{m}$ is indeed optically thin.

In summary, the important result obtained from these quantitative analyses is that the peak of the molecular gas in the central $1 \mathrm{kpc}$ of NGC 6240 is warm, dense, and moderately optically thin. The derived temperatures and densities of NGC 6240 are consistent with the high values found in the nearby starburst galaxies M82 and NGC 253 (Wild et al. 1992; J Jackson et al. 1995; Seaquist \& Fraver 2000). The low opacities predicted from these analyses are somewhat consistent with earlier results that investigate the opacities in M82 $(\tau=0.5-4.5$; Mao et al. 2000), but our new results predict much lower opacities than previously suggested in ULIRGs $(\tau=3-10$; Downes \& Solomon 1998). The low opacities found in the central region of NGC 6240 are possibly due to large velocity gradients caused by extremely turbulent gas, allowing emission in the core of the molecular cloud to escape efficiently. In addition, our predicted peak densities $\left(n_{\mathrm{H}_{2}}=10^{5.0-5.4} \mathrm{~cm}^{-3}\right)$ are much higher than the range of values found in ULIRGs using low $J$ transition CO emission $\left(n_{\mathrm{H}_{2}}=10^{2.3-4.3} \mathrm{~cm}^{-3}\right.$; Downes \& Solomon 1998). The disagreement is likely due to the difference in the excitation conditions of the observed molecular gas tracers; i.e. Downes \& Solomon (1998) used cold $(\mathrm{T} \sim 10 \mathrm{~K})$ and low density $\left(n_{\text {crit }} \sim 10^{3} \mathrm{~cm}^{-3}\right)$ tracers whereas our new observations trace much warmer $(\mathrm{T} \sim 30 \mathrm{~K})$ and denser $\left(n_{\text {crit }} \sim 10^{4} \mathrm{~cm}^{-3}\right.$ for $\mathrm{CO}(3-$ 2)) molecular gas. This study, therefore, demonstrates the importance of using high density submm line tracers such as $\mathrm{CO}(3-2)$ and $\mathrm{HCO}^{+}$emission to study the physical conditions in the nuclear ISM in ULIRGs.

\subsection{The Gas to Dust Mass Ratio}

The gas-to-dust mass ratio (i.e. $\mathrm{M}_{\mathrm{H}_{2}} / \mathrm{M}_{d}$ ) provides an important measure of the relative abundance between gas and metallicity. An average $\mathrm{M}_{\mathrm{H}_{2}} / \mathrm{M}_{d}$ over the entire galaxy is often derived in single dish work, where it is found that $\mathrm{M}_{\mathrm{H}_{2}} / \mathrm{M}_{d} \sim 100$ for Galactic sources (Hildebrand 1983), $\mathrm{M}_{\mathrm{H}_{2}} / \mathrm{M}_{d} \sim 200-300$ for local LIRGs/ULIRGs (Contini \& Contini 2003; Yao et al. 2003; Seaquist et al. 2004), and $\mathrm{M}_{\mathrm{H}_{2}} / \mathrm{M}_{d}=15-231$ in high- $z$ sources (Solomon \& vanden Bout 2005). Wilson et al. (2006 in prep) finds $\mathrm{M}_{\mathrm{H}_{2}} / \mathrm{M}_{d}=357 \pm 95$ from a sample of 13 LIRGs/ULIRGs observed in high resolution with the SMA. It is found that the global gas-to-dust mass ratio in NGC 6240 is $\mathrm{M}_{\mathrm{H}_{2}} / \mathrm{M}_{d}=(147 \pm 57$ ) (see $\S 3.3)$.

Our new high angular resolution $\mathrm{CO}(3-2)$ and $880 \mu \mathrm{m}$ maps allow us to study the spatial variation of $\mathrm{M}_{\mathrm{H}_{2}} / \mathrm{M}_{d}$. We have converted the $\mathrm{CO}(3-2)$ and $880 \mu \mathrm{m}$ maps to $\mathrm{M}_{\mathrm{H}_{2}}$ and $\mathrm{M}_{\mathrm{d}}$ maps. The gas-to-dust mass ratio map is shown in Figure [13. The peak of $\mathrm{M}_{\mathrm{H}_{2}} / \mathrm{M}_{d}$ occurs between the two AGNs, and near S2, suggesting an over abundance of dense gas relative to dust in these regions, assuming that most of the $880 \mu \mathrm{m}$ emission is tracing dust. The high ratios seen north of the northern AGN are due to low dust masses at the edge of the $3 \sigma$ cutoff. The peak ratio is $\sim 150$. We note that the ratio map obtained here is only available in the central $\mathrm{kpc}$ where the dust emission is significant $(>3 \sigma)$. The $\mathrm{CO}(3-2)$ emission is much more extended than the dust emission and hence the average $\mathrm{M}_{\mathrm{H}_{2}} / \mathrm{M}_{d}$ in Figure 13 is lower than the global $\mathrm{M}_{\mathrm{H}_{2}} / \mathrm{M}_{d}(147 \pm 57)$. Higher ratios are seen in the north-south direction (i.e. $\mathrm{M}_{\mathrm{H}_{2}} / \mathrm{M}_{d} \sim 100-160$ ) along the two AGNs than the east-west direction (i.e. $\left.\mathrm{M}_{\mathrm{H}_{2}} / \mathrm{M}_{d} \sim 30-100\right)$. The steep decline in the wings of dust emission may suggest substantial free-free emission associated with the starburst winds, or a local gradient in the metallicity.

\subsection{The FIR to CO (3-2) Luminosity Ratio}

It is empirically known that the ratio between FIR and CO (1-0) luminosities is higher for ULIRGs than for normal spiral galaxies (Solomon et al. 1997). Solomon et al. (1997) attribute this result to the presence of compact, optically thick dust emission, which in turn yields higher dust temperatures than in the optically thin limit. However, these measurements are performed using the CO (1-0) transition, and it is found that this value is different when the ratio between FIR and $\mathrm{CO}(3-$ 2 ) is taken (i.e. compare $\log \mathrm{L}_{\mathrm{FIR}} / \log \mathrm{L}_{\mathrm{CO}(3-2)}=$ $1.43 \pm 0.08$ with $\log \mathrm{L}_{\mathrm{FIR}} / \log \mathrm{L}_{\mathrm{CO}(1-0)}=1.72 \pm 0.21$; Yao et al. 2003). The FIR-to-CO (3-2) luminosity ratio appears to be even lower in high- $z$ sources where the FIR luminosity is typically an order of magnitude larger (i.e. $\mathrm{L}_{F I R} \sim 10^{13} \mathrm{~L}_{\odot}$ ). From the high$z$ sources with a robust $\mathrm{CO}(3-2)$ detection, the ratio is found to be $\log \mathrm{L}_{\mathrm{FIR}} / \log \mathrm{L}_{\mathrm{CO}(3-2)}=1.23 \pm 0.03$ (Solomon \& vanden Bout 2005; Iono et al. 2006). Using the CO (3-2) integrated intensity from our full resolution map, and the IRAS measurement for the FIR luminosity, the ratio for NGC 6240 is found to be $\log \mathrm{L}_{\mathrm{FIR}} / \log \mathrm{L}_{\mathrm{CO}(3-2)}=1.16$.

NGC 6240 thus contains much more molecular gas relative to the intensity of starburst activity than sim- 
ilarly FIR bright systems observed in the local universe. The ratio is even lower than the values derived in high$z$ sources. The overwhelming amount of molecular gas available in the central region of NGC 6240 suggests that there is enough gas to support star formation for at least another $\sim 10^{8}$ years, assuming that all of the gas will eventually be converted to stars with a constant star formation rate of $60 \mathrm{M}_{\odot} \mathrm{yr}^{-1}$. The wealth of molecular gas may also suggest that the star formation in NGC 6240 will increase to a level comparable to that seen in Arp 220.

\section{DISCUSSION}

\subsection{The Nature of the Western Molecular Complex} $(W C)$

Recent galaxy-galaxy collision simulations (e.g. Hopkins et al. 2005) that trace the evolution of the central AGN have shown that significant outflow activity may be observable in high $J$ CO transitions up to $J=6-5$ (Narayanan et al. 2006). While a massive $\left(3.5 \times 10^{8} \mathrm{M}_{\odot}\right)$ off-nuclear molecular complex such as WC is often explained by starburst superwinds (Heckman. Armus, \& Milev 1990), we investigate here the possibility that the origin of $\mathrm{WC}$ is gas entrained in outflow from the AGN of one of the progenitor galaxies. From an estimate of the cloud diameter of $D=2.7 \mathrm{kpc}$ from the $\mathrm{CO}(3-2)$ map and the velocity dispersion of $\sigma_{v}=400 \pm 100 \mathrm{~km} \mathrm{~s}^{-1}$ estimated from the FWHM of the $\mathrm{CO}(3-2)$ line in $\mathrm{WC}$, we find a virial mass of $\mathrm{M}_{\text {vir }}=3.0 \times 10^{11} \mathrm{M}_{\odot}$. The error in this virial mass estimate could be as large as a factor of (2-3) due to large uncertainties in the cloud size and velocity dispersion. By assuming the Galactic dynamical mass $\left(\mathrm{M}_{d y n}\right)$ to $\mathrm{M}_{\mathrm{H}_{2}}$ ratio of 136 (Nakanishi \& Sofue 2006), we estimate a total mass of $\mathrm{M}_{t o t}=4.8 \times 10^{10} \mathrm{M}_{\odot}$ for WC. Although the uncertainties in these measurements are large, the derived virial mass is an order of magnitude larger than the total mass of WC. This suggests that WC is not bound by its own gravitational force and will likely disperse within $D / \sigma_{v} \sim 7 \times 10^{6}$ years. The systemic velocity of WC is $v_{\text {sys }}=7449 \mathrm{~km} \mathrm{~s}^{-1}$, which yields a relative velocity $v_{\mathrm{WC}} \sim 210 \mathrm{~km} \mathrm{~s}^{-1}$ assuming that WC is ejected from the northern nucleus. Using a projected distance between the northern nucleus and WC of $4.4 \mathrm{kpc}$, the timescale for WC to traverse to the current location is $\sim 2 \times 10^{7}$ years.

Although all of these timescales contain significant uncertainties, the short dispersion time $\left(7 \times 10^{6}\right.$ years $)$ suggests that it is unlikely that the cloud would have survived the estimated transversal time of $2 \times 10^{7}$ years to reach the current location. Simulations conducted by Narayanan et al. (2006) predict that the total gas mass of an ejected cloud could be as large as $\sim 10^{8} \mathrm{M}_{\odot}$ with a cloud dispersion time of $t \sim 2.9 \times 10^{8}$ years (Narayanan et al. 2006). While the CO (3-2) derived gas mass is consistent with the ejected gas mass predicted from simulations, the derived dispersion timescale is a factor of 40 shorter. These results may suggest that the AGN outflow scenario is less appealing to explain $\mathrm{WC}$, and other scenarios such as molecular outflow from starburst superwinds may be more appealing to explain the large and extended molecular gas. The starburst outflow scenario to explain the western extension of ra- dio continuum emission in NGC 6240 was suggested by Colbert et al. (1994).

Possible scenarios to explain WC are not limited to starburst superwinds or to AGN outflows and both of the following are also possibilities; (1) molecular gas ejected from the violent arm-arm collision, (2) molecular gas complex formed locally as a result of gravitational instability. Ejection of molecular gas is possible as arm-arm collision could occur in each pericentric passage. However, ejecting a molecular gas mass of order $10^{8} \mathrm{M}_{\odot}$ and diameter $1-2 \mathrm{kpc}$ from the potential of a tidal arm may require a significant amount of energy. While it is possible that gravitational collapse could form a $\mathrm{CO}$ cloud from an ambient $\mathrm{H}$ I cloud, it is already demonstrated that the virial mass is an order of magnitude larger than the estimated total mass of WC. Hence these two scenarios are less appealing to explain the origin of WC than the starburst superwind or the AGN outflow scenarios. Our current data, however, is not sensitive enough to address the exact origin of $\mathrm{WC}$, and future observations with higher angular resolution and sensitivity should provide us further understandings to the possibilities of these scenarios.

\subsection{The Central 1 kpc of NGC 6240}

Our new analysis presented in $\S 4.1$ shows that the molecular gas in the central $1 \mathrm{kpc}$ of NGC 6240 is warm, dense and moderately optically thin. These results suggest that the excitation conditions of molecular gas surrounding the two AGNs is similar to the nuclear regions of nearby starbursting galaxies. The low opacity is likely caused by highly turbulent gas, which is also supported by the dominance of non-circular kinematics entangled with possible disk-like rotation around the northern nucleus (see §3.1.2). The southern nucleus shows little evidence of disk-like rotation possibly due to the dominance of non-circular motion, or due to a face-on orientation of a rotating disk. Although the projected nuclear separation is different, the double nucleus configuration is similar to that seen in Arp 220 where a merger of two counter-rotating disks is suggested from high resolution CO (2-1) observations (Sakamoto et al. 1999). Higher angular resolution observations should provide further insight to these possibilities.

In addition to the large amount of dense molecular gas, there is significant multi-wavelength evidence that the central $1 \mathrm{kpc}$ also harbors substantial amounts of dust. While we caution here that emission traced in $880 \mu \mathrm{m}$ and $1.3 \mathrm{~mm}$ (Tacconi et al. 1999) are possibly more dominated by non-thermal emission, the ubiquitous presence of the optically thin dust emission in the medium between the two AGNs is a significant evidence of cold dust. Further, from comparison between star formation activity traced in optical and the AGN activity traced in X-ray, Gerssen et al. (2004) have shown that the two nuclear star forming regions in both progenitor disks are not coincident with the AGN positions but are offset by $\sim 0$ "' 5 , suggesting that foreground dust extinction in the central region is significant. The dusty central $1 \mathrm{kpc}$ also shows significant evidence of a coexistence of hot and shocked gas as seen in detections of maser emission (Hagiwara. Diamond \& Mivoshi 2003) and $\mathrm{H}_{2}$ emission (Ohyama et al. 2000). Both of these are detected near S1 and S2, providing evidence of shocked gas asso- 
ciated with either the AGN jet or the nuclear starburst activity. Summarizing the multi-wavelength properties, the central $1 \mathrm{kpc}$ of NGC 6240 harbors two AGNs surrounded by a wealth of warm and dense molecular gas, as well as hot ( $\left.\mathrm{T}=10^{(3-7)} \mathrm{K}\right)$ gas and a large amount of dust, and the region is undergoing a high rate of star formation activity.

\section{SUMMARY}

We present high resolution $\mathrm{CO}(3-2), \mathrm{HCO}^{+}(4-3)$ and $880 \mu \mathrm{m}$ imaging of the luminous infrared galaxy NGC 6240 obtained at the SMA. We provide our main findings below;

1. The distribution of $\mathrm{CO}(3-2)$ is extended on a $4 \mathrm{kpc}$ scale, but the $\mathrm{HCO}^{+}(4-3)$ emission is more compact and concentrated in the central $1 \mathrm{kpc}$ between the two nuclear AGNs. The $\mathrm{CO}(3-2), \mathrm{HCO}^{+}(4-$ 3 ), and $880 \mu \mathrm{m}$ emission all peak between the two AGNs where the $\mathrm{H}_{2}$ emission was detected previously.

2. The kinematical information provided by the CO (3-2) emission shows a rotating disk-like feature centered around the northern AGN, but the kinematics of gas between the two nuclei is extremely turbulent.
3. Our LVG analysis shows that the gas near the peak of the continuum emission is warm $(\mathrm{T}=20$ $-100 \mathrm{~K})$, dense $\left(n_{\mathrm{H}_{2}}=10^{5.0-5.4} \mathrm{~cm}^{-3}\right)$ and moderately optically thin $(\tau=0.2-2)$ in the central $1 \mathrm{kpc}$ of NGC 6240.

4. We propose that the isolated molecular emission with $3.5 \times 10^{8} \mathrm{M}_{\odot}$ seen west of the main $\mathrm{CO}$ component may be associated with outflows from starburst superwinds, but the gas outflow scenario from one of the central AGN is not completely ruled out.

We thank Kouichiro Nakanishi, Sachiko Okumura and Naomasa Nakai for providing their published CO (1$0)$ and $\mathrm{HCO}+(1-0)$ data cubes. We also thank Linda Tacconi for kindly providing us with the CO (2-1) data cube. The Submillimeter Array is a joint project between the Smithsonian Astrophysical Observatory and the Academia Sinica Institute of Astronomy and Astrophysics, and is funded by the Smithsonian Institution and the Academia Sinica.

\section{REFERENCES}

Aalto, S., Booth, S. R., Black, J. H., \& Johansson, L. E. B. 1995, å, 300,369

Armus, L., Heckman, T., \& Miley, G. 1987, AJ, 94, 831

Armus et al., 2006, ApJ, 640, 204

Barnes, J. E., \& Hernquist, L. 1996, ApJ, 370, L65

Bergin, E. A., \& Langer, W. D. 1997, 486, 316

Beswick, R. J., Pedlar, A., Mundell, C. G., \& Gallimore, J. F. 2001, MNRAS, 325, 151

Blake, G. A., Sutton, E. C., Masson, C. R., \& Phillips, T. G. 1987, ApJ, 315, 621

Bryant, P. M., \& Scoville, N. Z. 1999, AJ, 117, 2632

Bogdanovic, T., Ge, J., Max, C. E., \& Raschke, L. M. 2003, AJ, 126, 2299

Boller, Th., Keil, R., Costantini, E., Fujimoto, R., Anabuki, N., Lehmann, I., \& Gallo, L. 2003, å, 411, 63

Bushouse, H. A., Borne, K. D., Colina, L., Lucas, R. A., Rowan-Robinson, M., Baker, A. C., Clements, D. L., Lawrence, A., \& Oliver, S. 2002, ApJS, 138, 1

Colbert, E. J. M., Wilson, A. S. \& Bland-Hawthorn, J. 1994, ApJ, 436, 89

Contini, M., \& Contini, T. 2003, MNRAS, 342, 299

Di Matteo, T., Springel, V., \& Hernquist, L. 2005, Nature, 433, 604

Downes, D., \& Solomon, P. M. 1998, ApJ, 507, 615

Egami, E., Neugebauer, G., Soifer, B. T., Mathews, K., Becklin, E. E. \& Ressler, M. E. 2006, AJ, 131, 1253

Farrah, D., Rowan-Robinson, M., Oliver, S., Serjeant, S., Borne, K., Lawrence, A., Lucas, R. A., Bushouse, \& H., Colina, L. 2001, MNRAS, 326, 1333

Flower, D. R., \& Launay, J. M. 1985, MNRAS, 214, 271

Flower, D. R. 1999, MNRAS, 305, 651

Gallimore, J. F., \& Beswick, R. 2004, AJ, 127, 239

Gao, Y., \& Solomon, P. M. 2004, ApJ, 152, 63

Gerssen, J., van der Marel, P. V., Axon, D., Mihos, C. J.,

Hernquist, L., \& Barnes, J. 2003, AJ, 127, 75

Genzel, R., et al., 1998, ApJ, 498, 579

Goldreich, P. \& Kwan, J. 1974, 189, 441

Greve, T. R., et al. 2005, MNRAS, 359, 1165

Greve, T. R., Papadopoulos, P. P., Gao, Y., \& Radford, S. J. E. 2006, ApJ submitted (astro-ph/0610378)
Ikebe, Y., Leighly, K., Tanaka, Y., Nakagawa, T., Terashima, Y., \& Komossa, S. 2000 MNRAS, 316, 433

Hagiwara, Y., Diamond, P. J., \& Miyoshi, M. 2003, å, 400, 457

Heckman, T. M., Armus, L., \& Miley, G. K. 1990, ApJS, 74, 833

Hildebrand, R. H. 1983, QJRAS, 24, 267

Ho, P. T. P., Moran, J. M., \& Lo, K. Y. 2004, ApJ, 616, L1

Hopkins, P. F., Hernquist, L., Cox, T. J., Di Matteo, T., Martini, P., Robertson, B., \& Springel, V. 2005, ApJ, 630, 705

Hopkins, P. F., Hernquist, L., Cox, T. J., Di Matteo, T., Robertson, B., \& Springel, V. 2006, ApJS, 163, 1

Imanishi, M., Dudley, C. C., \& Maloney, P. R. 2006, ApJ, 637, 114

Iono, D., Yun, M. S., \& Mihos, C. J. 2004, ApJ, 616, 199

Iono, D., Ho, P. T. P., Yun, M. S., Matsushita, S., Peck, A. B., \& Sakamoto, K. 2004, ApJ, 616, L63

Iono, D., Tamura, Y., Nakanishi, K., Kawabe, R., Kohno, K. Okuda, T., Yamada, K., Hatsukade, B., \& Sameshima, M. 2006, PASJ, 58, 6 in press

Jackson, J. M., Paglione, T. A. D., CArlstrom, J. E. \& Ngueyn, Q. R. 1995, ApJ, 438, 695

Kennicutt, R. C. 1998, ApJ, 498, 541

Klaas, U., Haas, M., Muller, S. A. H., Chini, R., Schulz, B., Coulson, I., Hippelein, H., Wilke, K., Albrecht, M., \& Lemke, D. 2001, à 379,823

Komossa, S., Burwitz, V., Hasinger, G., Predehl, P., Kaastra, J. S., \& Ikebe, Y. 2003, ApJ, 582, L15

Laurent-Muehleisen, S. A., Kollgaard, R. I., Ryan, P. J., Feigelson, E. D., Brinkmann, W., \& Siebert, J. 1997, A\&A, 122,235

Lonsdale, C. J., Farrah, D. \& Smith, H. E., 2006, "Astrophysics Update 2 - topical and timely reviews on astronomy and astrophysics". Ed. John W. Mason. Springer/Praxis books.

Lutz, D., Sturm, E., Genzel, R., Spoon, H. W. W., Moorwood, A.

F. M., Netzer, H., \& Sternberg, A. 2003, å, 409, 867

Matsushita, S., Kohno, K., Vila-Vilaro, B., Tosaki, T., \&

Kawabe, R. 1998, ApJ, 495, 267

Mao, R. Q., Henkel, C., Schulz, A., Zielinsky, M., Mauersberger, R., Storzer, H., Wilson, T. L., \& Gensheimer, P. 2000, å, 358, 433 
Max, C. E., Canalizo, G., Macintosh, B. A., Raschke, L., Whysong, D., Antonucci, R., \& Schneider, G. 2005, ApJ, 621, 738

Mihos, C. J., \& Hernquist, L. 1996, ApJ, 464, 641

Nakanishi, K., Okumura, S. K., Kohno, K., Kawabe, R., \& Nakagawa, T. 2005, PASJ, 57, 575

Nakanishi, H., \& Sofue, Y. 2006, PASJ, 58, 847

Narayanan, D., Cox, T. J., Robertsonm, B., Dave, R., Di Matteo, T., Hernquist, L., Hopkins, P., Kulesa, C., Walker, C. ApJin press (astro-ph/060474)

Netzer, H., Lemze, D., Kaspi, S., George, I. M., Turner, T. J., Lutz, D., Boller, T., \& Chelouche, D. 2005, ApJ, 629, 739

Ohyama, Y., Yoshida, M., \& Takata, T. 2003, AJ, 126, 229

Ohyama, Y. et al., 2000, PASJ, 52, 563

Pasquali, A., Gallagher, J. S., \& de Grijs, R. 2004, å, 415, 103

Petitpas, G. R., \& Wilson, C. D. 2000, ApJ, 538, 117

Sakamoto, S., Hayashi, M., Hasegawa, T., Handa, T., \& Oka, T. 1994, ApJ, 425, 641

Sakamoto, K., Scoville, N. Z., Yun, M. S., Crosas, M., Genzel, R., \& Tacconi, L. J. 1999, ApJ, 514, 68

Sanders, D. B., \& Mirabel, I. F. 1996, ARA\&A, 34, 749

Scoville, N. Z., \& Sanders, D. B. 1987, in Interstellar Processes (Dordrecht: Reidel), 21

Scoville, N. Z., et al. 2000, AJ, 119, 991

Seaquist, E. R. \& Frayer, D. T. 2000, ApJ, 540, 765
Seaquist, E. R., Yao, L., Dunne, L. Cameron, H. 2004, MNRAS, 349,1428

Solomon, P. M., Downes, D., Radford, S. J. E., \& Barrett, J. W. 1997, ApJ, 478, 144

Solomon, P. M., \& vanden Bout, P. A. 2005, ARA\&A, 478, 144

Springel, V., Di Matteo, T., \& Hernquist, L. 2005, MNRAS, 361, 776

Surace, J. A., Sanders, D. B., Vacca, W. D., Veilleux, S., \& Mazzarella, J. M. 1998, ApJ, 492, 116

Tacconi, L. J., Genzel, R., Tecza, M., Gallimore, J. F., Downes, D., \& Scoville, N. Z. 1999, ApJ, 524, 732

Tacconi, L. J., et al. 2006, ApJ, 640, 228

Takakuwa, S., Mikami, H., \& Saito, M. 1998, ApJ, 501, 723

Wang, J., Zhang, Q., Zhong, W., Ho, P. T. P., Fazio, G., \& Wu, Y. 2004, ApJ, 616, L67

Wild, W., et al., 1992, A\&A, 265, 447

Yao, L., Seaquist, E. R., Kuno, N., \& Dunne, L. 2003, ApJ, 588, 771

Young, J. S., Kenney, J. D., Tacconi, L., Claussen, M. J., Huang, Y. -L., Tacconi-Garman, L., Shuding, X., \& Schloerb, F. P. 1986, ApJ, 311, L17

Yun, M. S., \& Carilli, C. 2002, ApJ, 568, 88 
TABLE 1

Derived Properties

\begin{tabular}{|c|c|c|}
\hline Observation & Property & Value \\
\hline \multicolumn{3}{|l|}{$\mathrm{CO}(3-2)$} \\
\hline & $v_{\text {sys }}\left(\mathrm{km} \mathrm{s}^{-1}\right)$ & $7449 \pm 25$ \\
\hline & FWZI $\left(\mathrm{km} \mathrm{s}^{-1}\right)$ & $1175 \pm 25$ \\
\hline & $\mathrm{S}_{\nu} \mathrm{d} \nu\left(\mathrm{Jy}_{\mathrm{km} \mathrm{s}}{ }^{-1}\right)$ & $2834 \pm 20$ \\
\hline & peak R.A. & $16^{\mathrm{h}} 52^{\mathrm{m}} 58.9^{\mathrm{s}}$ \\
\hline & peak Decl. & $2^{\circ} 24^{\prime} 03^{\prime \prime} 7$ \\
\hline & peak $\mathrm{T}_{B}(\mathrm{~K})$ & 8.6 \\
\hline \multicolumn{3}{|l|}{$\mathrm{HCO}^{+}(4-3)$} \\
\hline & $v_{\text {sys }}\left(\mathrm{km} \mathrm{s}^{-1}\right)$ & $7339 \pm 50$ \\
\hline & $\mathrm{FWZI}^{1}\left(\mathrm{~km} \mathrm{~s}^{-1}\right)$ & $300 \pm 50$ \\
\hline & $\mathrm{S}_{\nu} \mathrm{d} \nu^{1}\left(\mathrm{Jy}_{\mathrm{km} \mathrm{s}}{ }^{-1}\right)$ & $28 \pm 4$ \\
\hline & peak R.A. & $16^{\mathrm{h}} 52^{\mathrm{m}} 58.9^{\mathrm{s}}$ \\
\hline & peak Decl. & $2^{\circ} 24^{\prime} 03^{\prime \prime}{ }^{\prime} 8$ \\
\hline \multirow{5}{*}{$880 \mu \mathrm{m}$ continuum } & peak $\mathrm{T}_{B}(\mathrm{~K})$ & 0.5 \\
\hline & $\mathrm{S}_{880}(\mathrm{mJy})$ & $105 \pm 6$ \\
\hline & peak R.A. & $16^{\mathrm{h}} 52^{\mathrm{m}} 58.9^{\mathrm{s}}$ \\
\hline & peak Decl. & $2^{\circ} 24^{\prime} 03^{\prime \prime} .4$ \\
\hline & peak $\mathrm{T}_{B}(\mathrm{~K})$ & 0.1 \\
\hline
\end{tabular}

1 The FWZI and $\mathrm{S}_{\nu} \mathrm{d} \nu$ of the $\mathrm{HCO}^{+}(4-3)$ emission may be underestimated since the emission occurs near the edge of the bandpass (see text). 


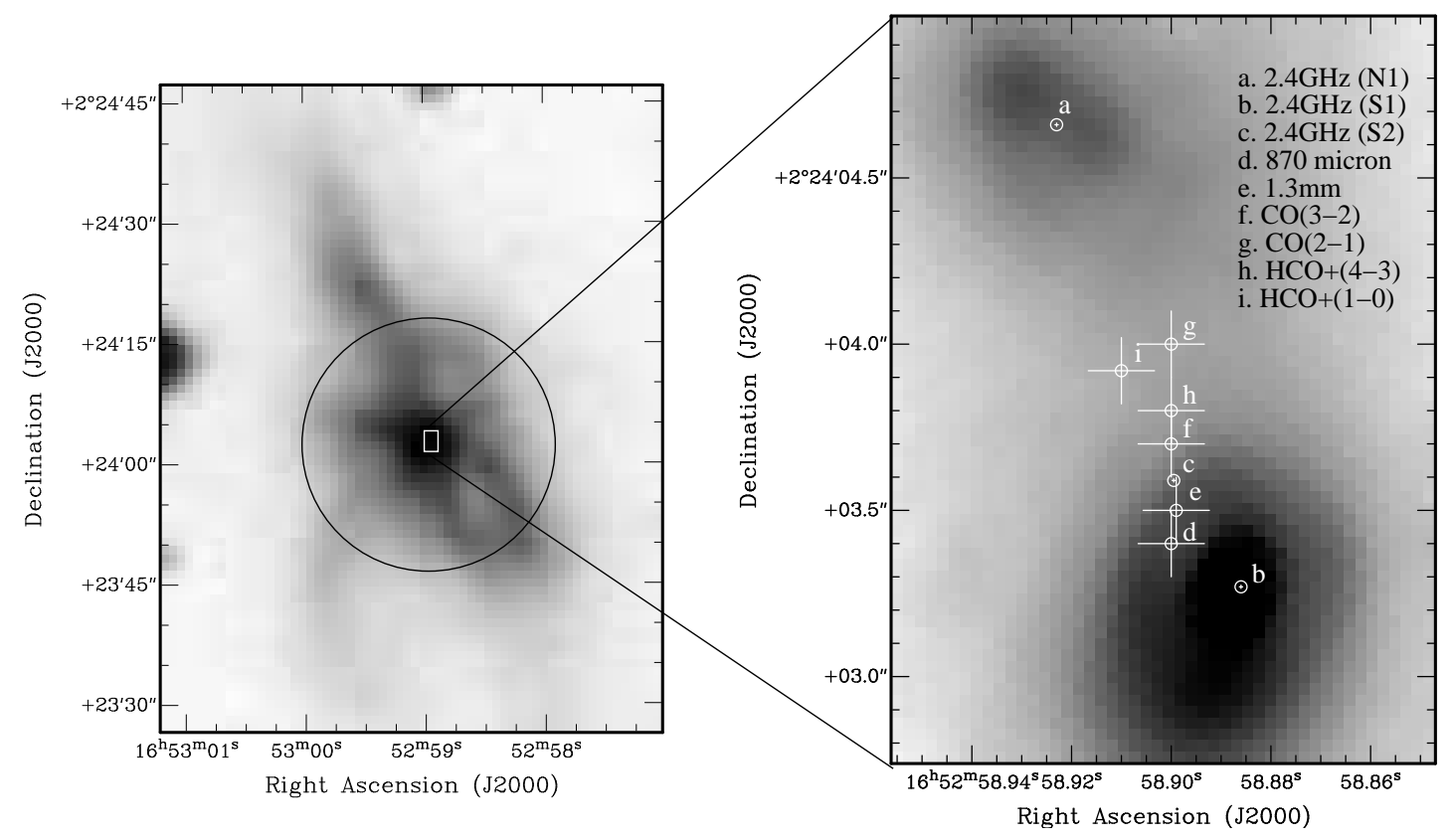

FIG. 1.- (left) Digital Sky Survey image of NGC 6240 overlaid with the 35" SMA field of view. (right) The HST NICMOS $2.2 \mu \mathrm{m}$ image of the central $2^{\prime \prime}(1 \mathrm{kpc})$ of NGC 6240 in grayscale overlaid with various peak positions from radio/mm/submm observations. Since accurate astrometry is not provided in the HST image, we align the peak of the southern $2.2 \mu \mathrm{m}$ nucleus with the $2.4 \mathrm{GHz}$ nucleus. The length of the crosses indicate the astrometric uncertainties of each measurement. The points labeled $\mathrm{a}$ and $\mathrm{b}$ are the locations of the two AGNs. Coordinates are obtained from (a,b,c) Gallimore \& Beswick (2004), (d,f,h) this work, (e,g) Tacconi et al. (1999), and (i) Nakanishi et al. (2005).
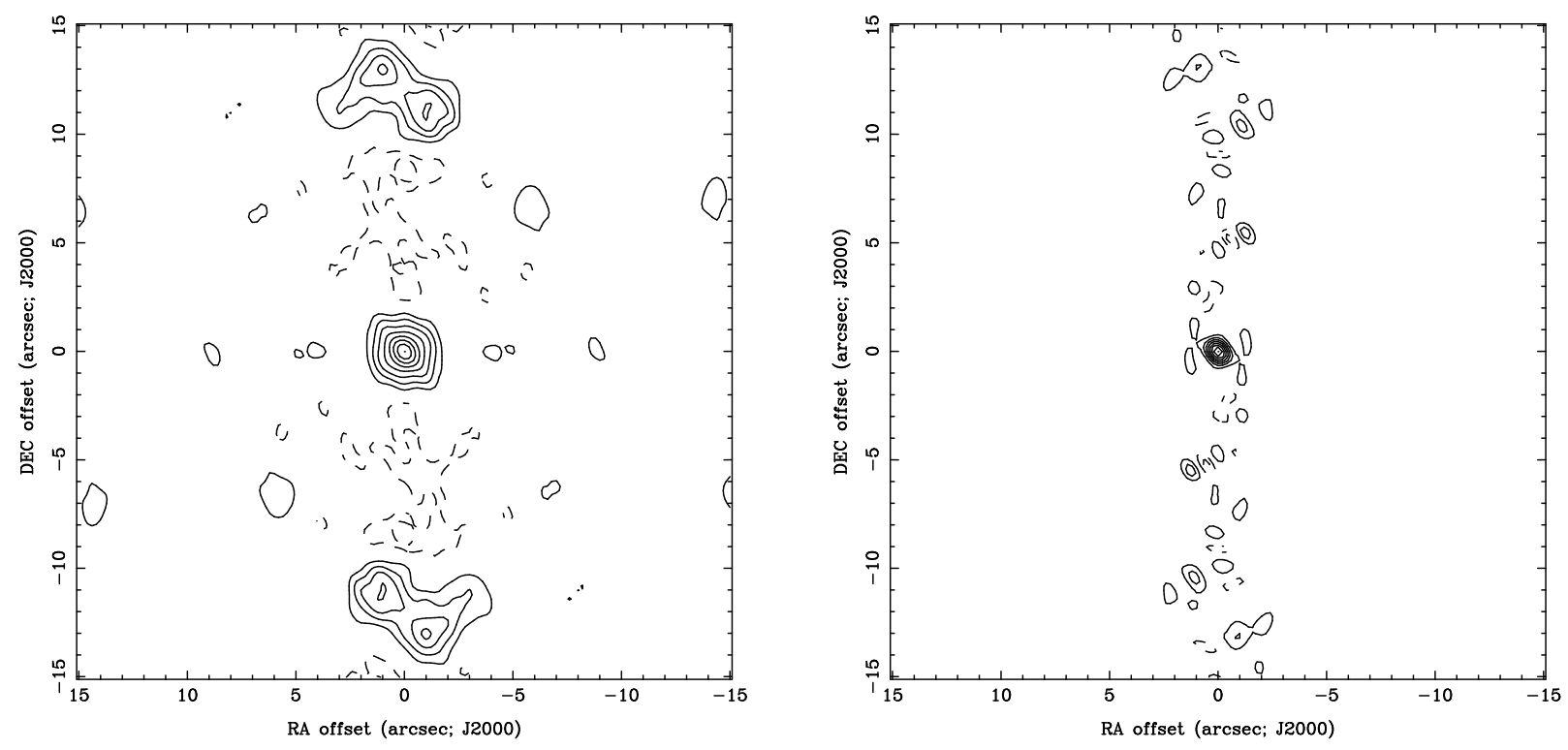

FIG. 2.- The synthesized beam using (left) natural and (right) uniform weighting. The contour levels are $10-90 \%$ of the peak. 


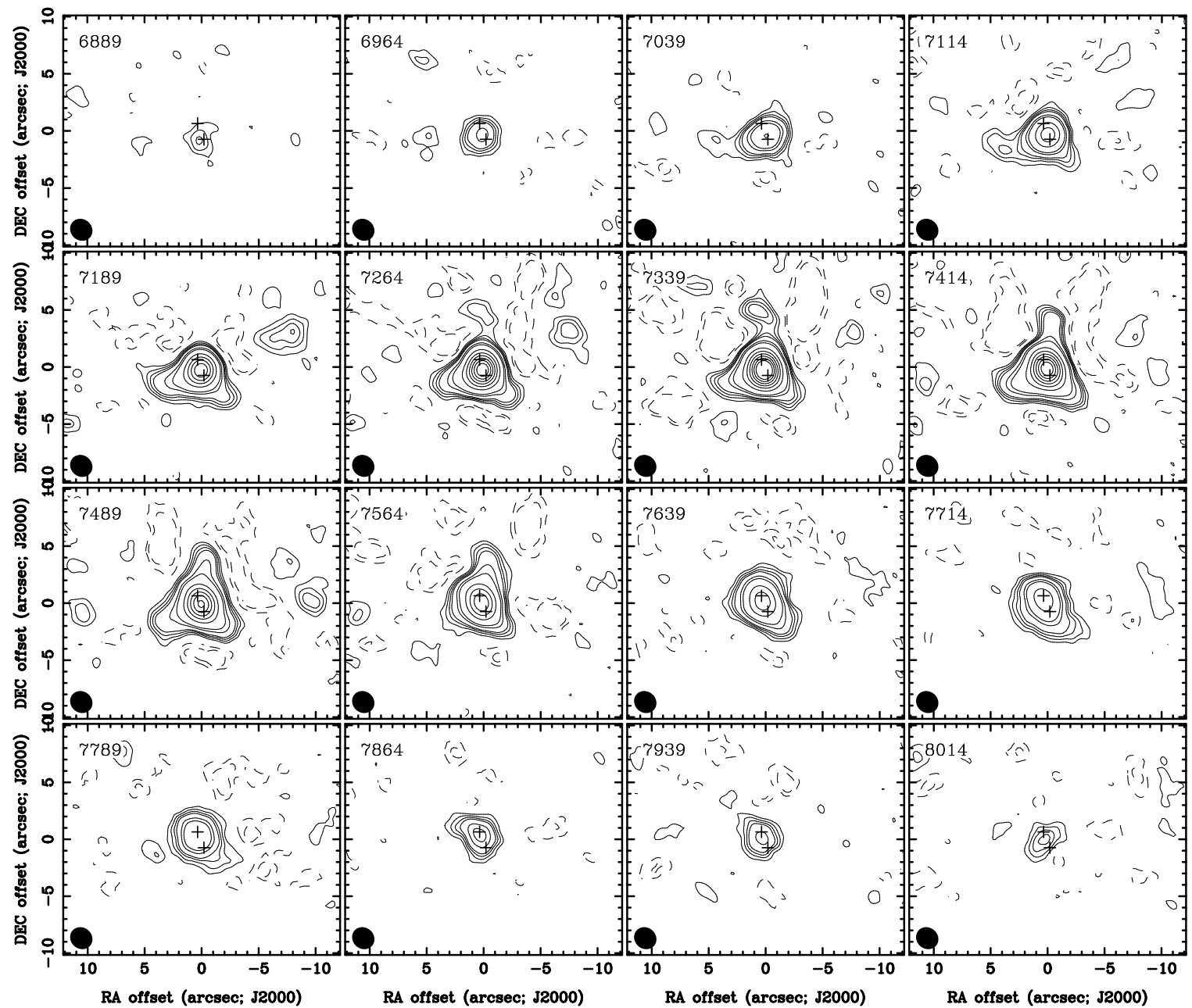

Fig. 3. - The natural weighted CO (3-2) channel maps in the velocity range 6889 to $8014 \mathrm{~km} \mathrm{~s}^{-1}$. Although the original channel maps were made with $25 \mathrm{~km} \mathrm{~s}^{-1}$ velocity resolution, the maps presented here are made by averaging three channels $\left(75 \mathrm{~km} \mathrm{~s} \mathrm{~s}^{-1}\right)$ in order to present the extremely broad velocity width in a single figure. The velocities are shown in the upper left corner of each panel, and the synthesized beam is shown in the lower left. The two crosses show the location of the two AGNs (N1 and S1 in Figure 1). The contour levels are 16 mJy (1 sigma) $(\times-5,-3,3,5,7,9,15,20,40,60,80,100,120,140)$.
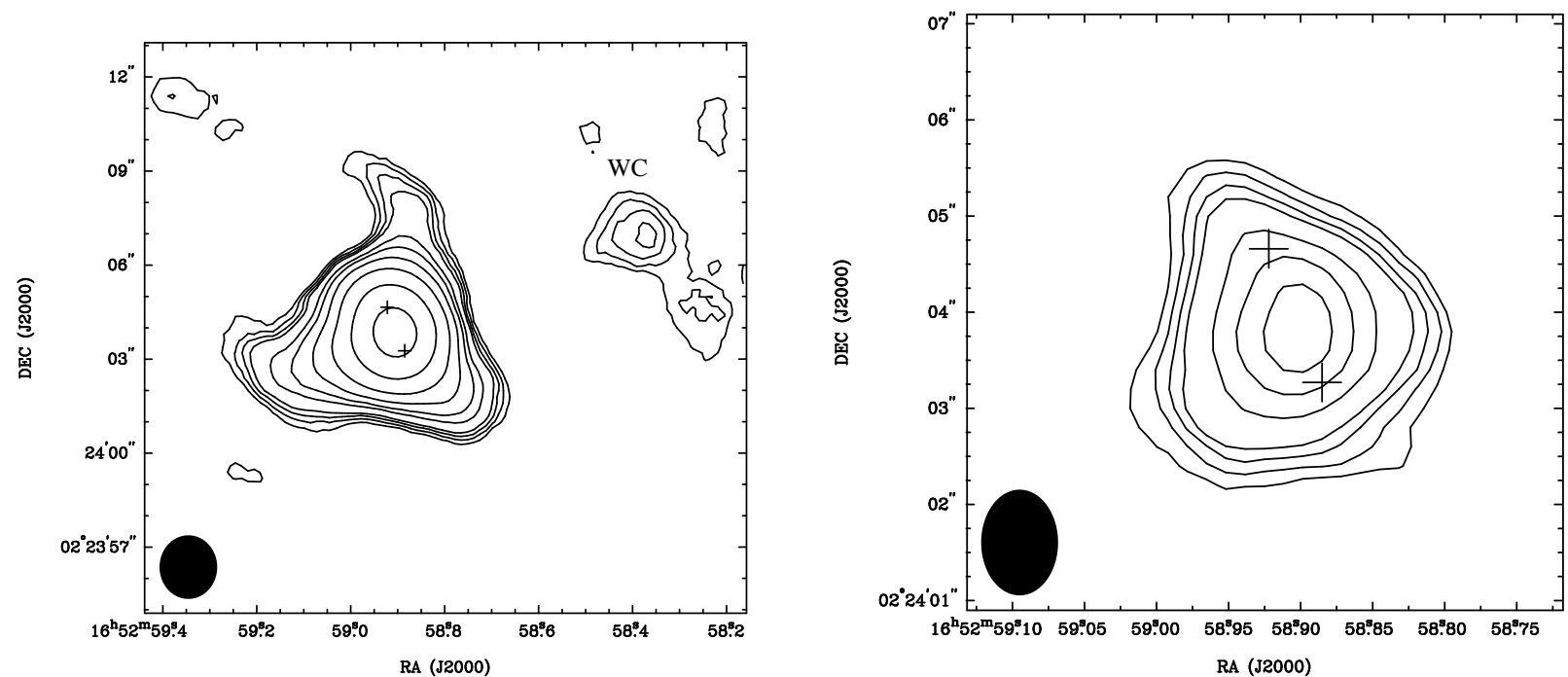

FIG. 4.- (left) The CO (3-2) moment 0 map of the central $18^{\prime \prime}$ of NGC 6240 . The contour levels are $5.5 \mathrm{Jy} k m \mathrm{~s}^{-1}(\times$ $3,5,7,9,15,20,30,50,100,200)$. We define the isolated CO (3-2) emission complex west of the main CO (3-2) concentration as WC. (right) The uniformly weighted CO (3-2) moment 0 map of the central $6^{\prime \prime}$ of NGC 6240 . The contour levels are $16 \mathrm{Jy} \mathrm{km} \mathrm{s}^{-1}(\times 3,5,7,9,15,21,27)$. The crosses are the locations of N1 and S1 (see Figure1), and the synthesized beams are shown in the lower left. 

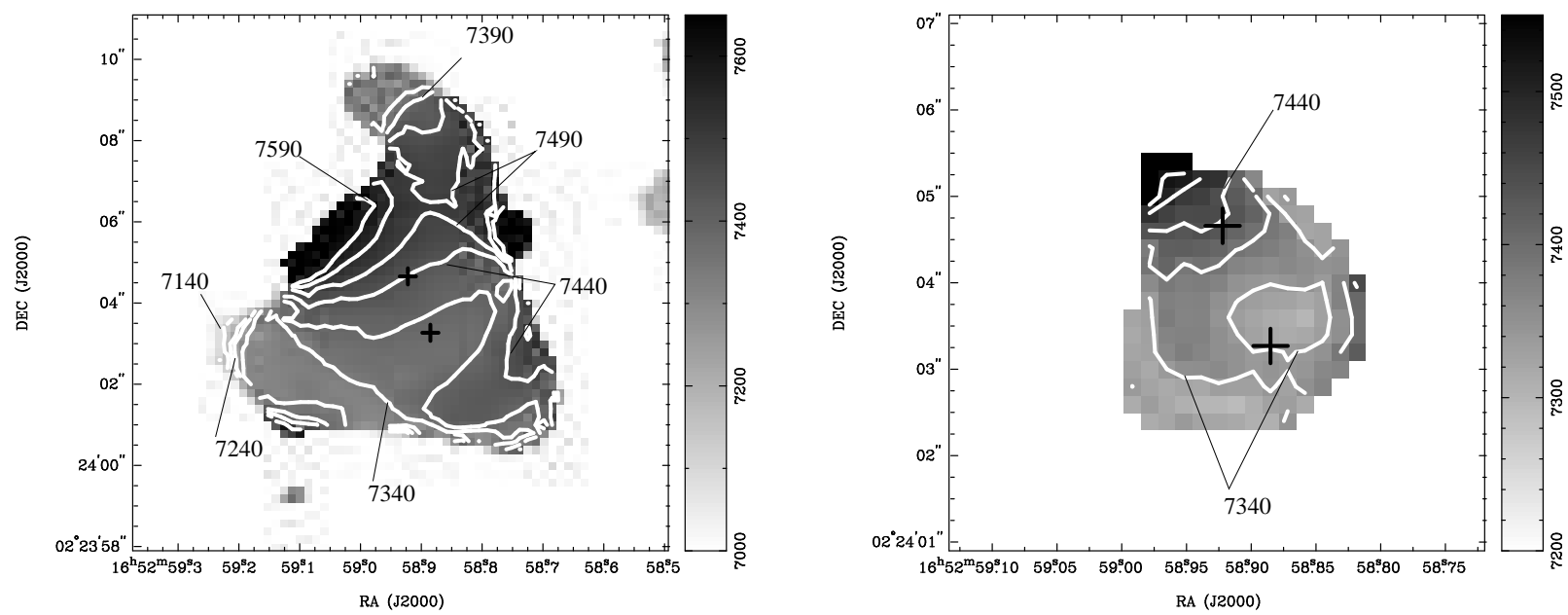

Fig. 5.- (left) The CO (3-2) velocity distribution (moment 1) map from the natural weighted map in the central $14^{\prime \prime}$ of NGC 6240. The velocity contours are spaced at $50 \mathrm{~km} \mathrm{~s}^{-1}$. (right) same as left but using uniform weighting. The corresponding moment 0 maps are shown in Figure 4. The units are in $\mathrm{km} \mathrm{s}^{-1}$.

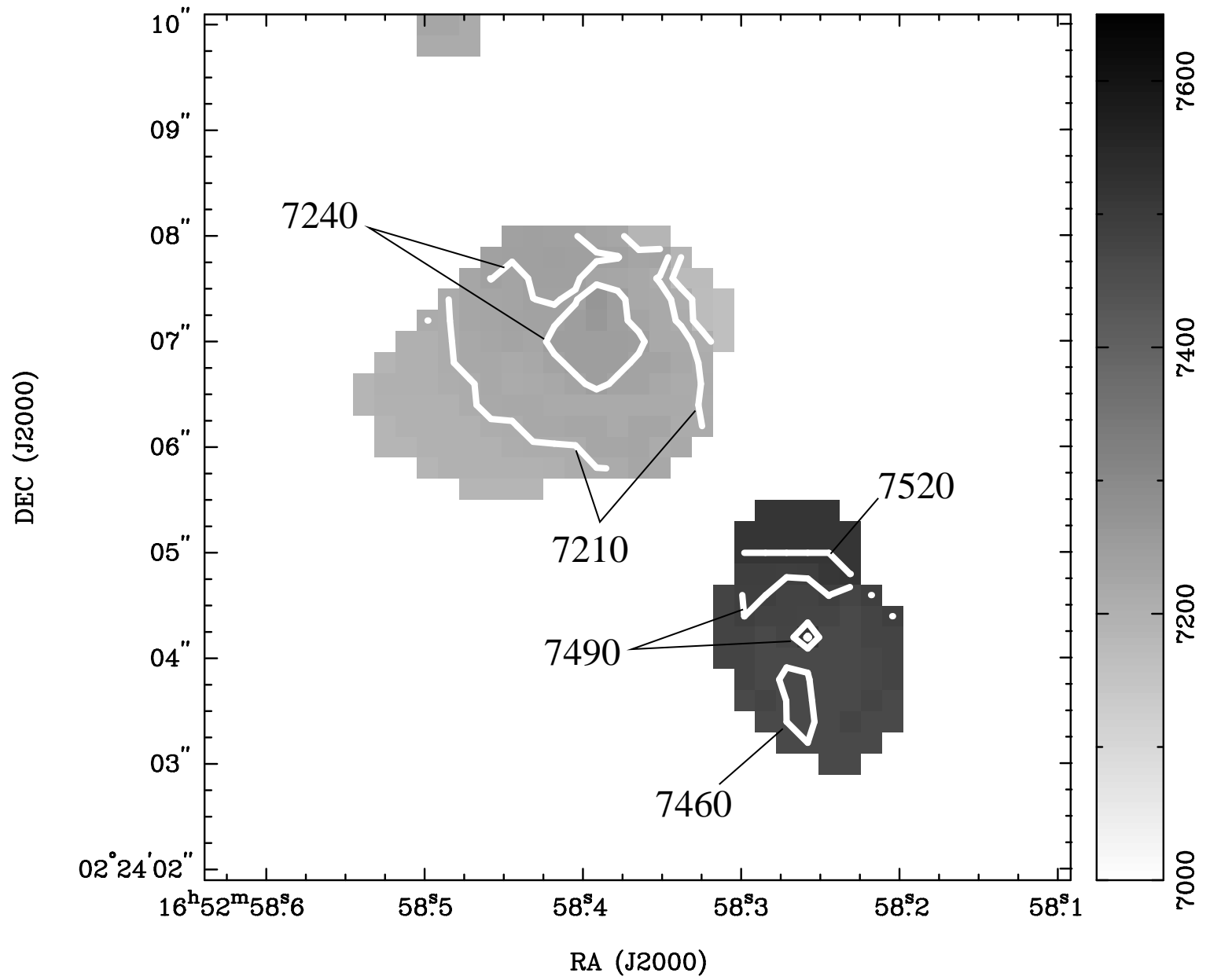

FIG. 6. - The CO (3-2) velocity distribution of the western complex (WC) alone. The velocity contours are spaced at $30 \mathrm{~km} \mathrm{~s}^{-1}$. The units are in $\mathrm{km} \mathrm{s}^{-1}$. 

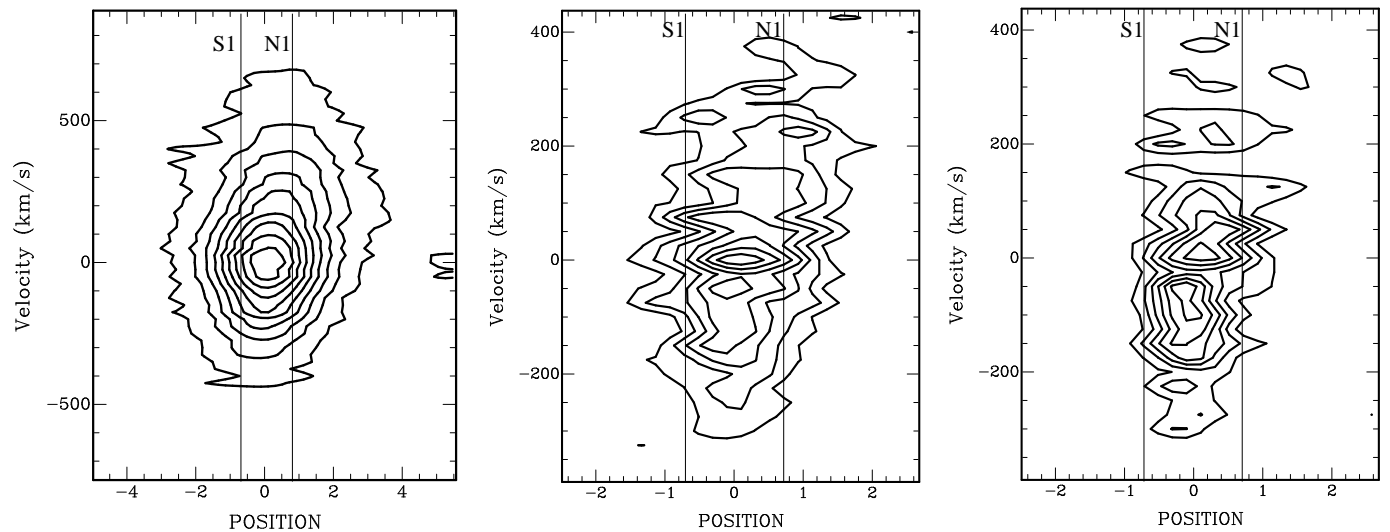

FIG. 7. - The position velocity diagram for (left) the $2{ }^{\prime \prime} 0 \times 11^{\prime \prime} 8$ resolution map (natural weighting). The x-axis shows the offset position from the center in arcseconds, and the y-axis shows the velocity in $\mathrm{km} \mathrm{s}^{-1}$. The contours represent $10,20,30,40,50,60,70,80,90 \%$ of the peak. These PVDs are made by slicing the cube along the two AGNs (N1 and S1) and centered exactly halfway between the two AGNs. The location of the two AGNs are shown as vertical lines. The linewidth near the two nuclei is extremely large, with emission slightly lopsided toward the redshifted velocity. (middle) Similar to left but for the $1^{\prime \prime} 1 \times 00^{\prime \prime} 8$ resolution map (uniform weighting). The contours represent the $1,5,10,20,40,60,80 \%$ of the peak. and (right) $0.0^{\prime \prime} 9 \times 0 . .5$ resolution map (using the longest baselines only). The contours represent the $15,30,45,60,75,90 \%$ of the peak. The nuclear region is resolved into two distinct components in the highest resolution map.
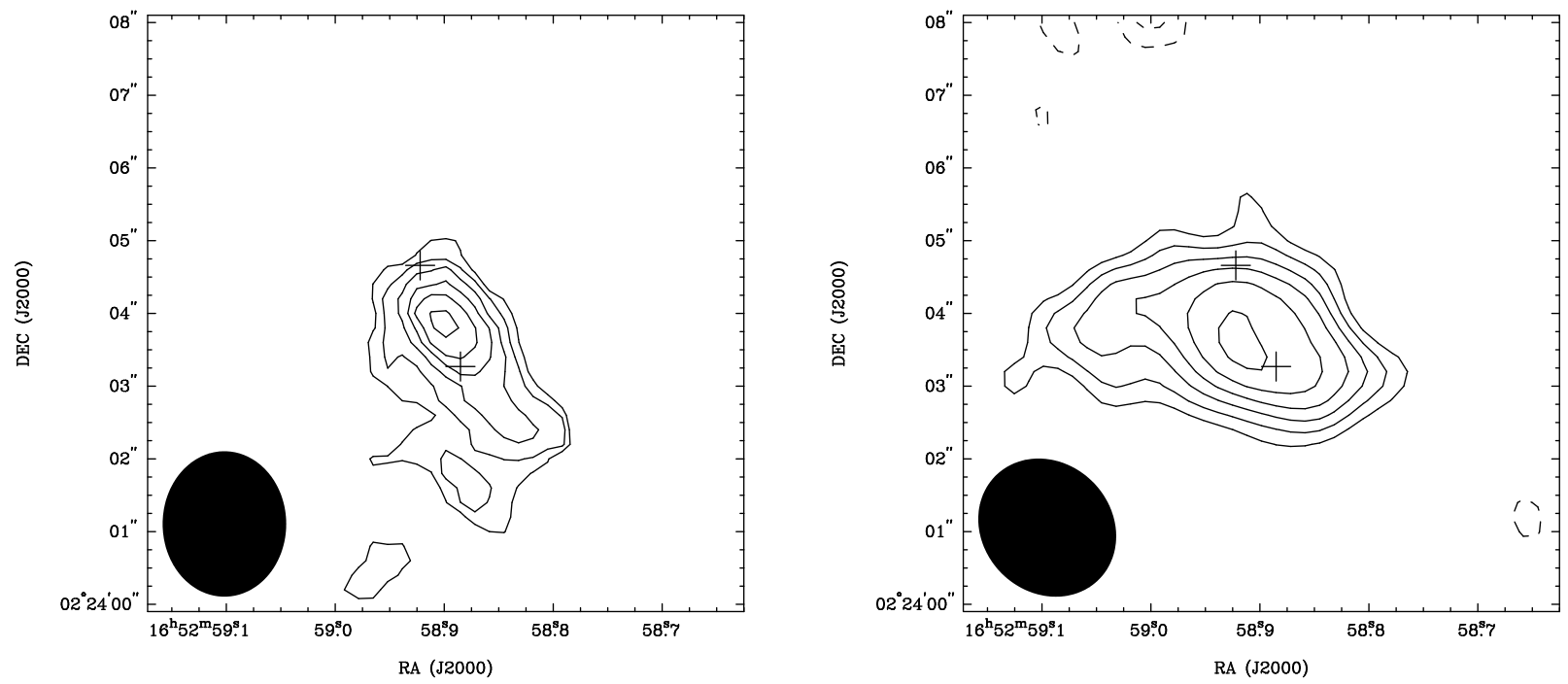

FIG. 8. - (left) The integrated intensity map of the $\mathrm{HCO}^{+}(4-3)$ emission. The contours are $5 \mathrm{Jy} \mathrm{km} \mathrm{s}{ }^{-1}(\times 3,4,5,6,7,8)$. (right) The $880 \mu \mathrm{m}$ emission map. The contours are $6 \mathrm{mJy}(\times-4,-3,3,4,5,6,7)$. Both of these maps are made using natural weighting, and the symbols in both maps are the same as in Figure 4 


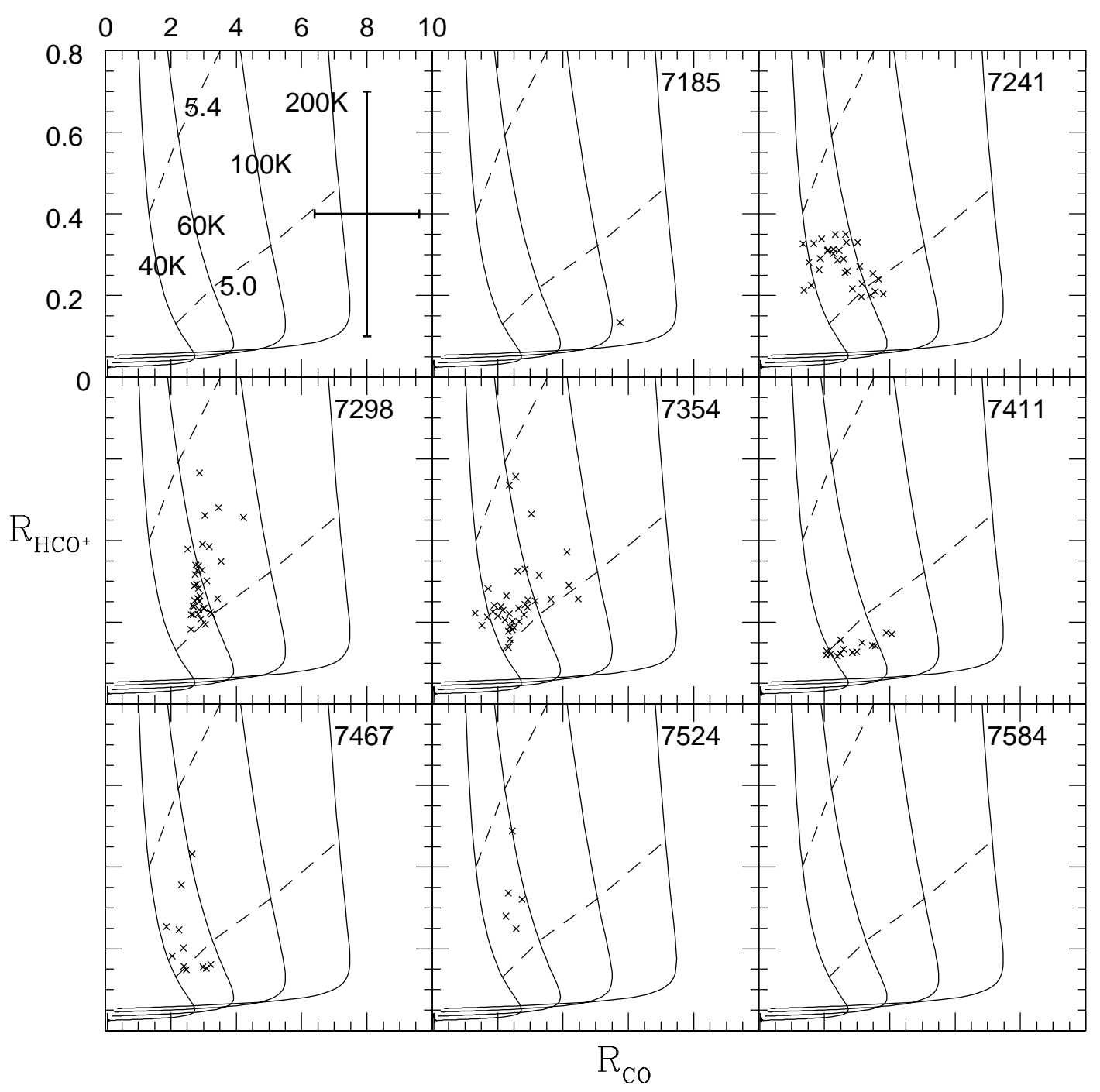

Fig. 9. - The $\mathrm{R}_{C O}(\mathrm{CO}(3-2) / \mathrm{CO}(1-0))$ vs. $\mathrm{R}_{H C O}+\left(\mathrm{HCO}^{+}(4-3) / \mathrm{HCO}^{+}(1-0)\right)$ plot for each $56 \mathrm{~km} \mathrm{~s}^{-1}$ channel. The crosses show the pixel-to-pixel correlation of the two ratios. The cross shown in the lower right of the first panel represents a typical error bar associated with the data. $\mathrm{X}_{C O} /(\mathrm{dv} / \mathrm{dr})=10^{-7}\left(\mathrm{~km} \mathrm{~s}^{-1} \mathrm{pc}^{-1}\right)^{-1}$ is adopted for this particular model. The solid line shows the temperature contours of $40,60,100$, and $200 \mathrm{~K}$, and the dashed line shows density contours of $10^{5.0}$ and $10^{5.4} \mathrm{~cm}^{-3}$. The lower density contours (i.e. $10^{4.0} \mathrm{~cm}-3$ ) lie in the lower left corner of each panel, which are not visible with the current scaling. 
16
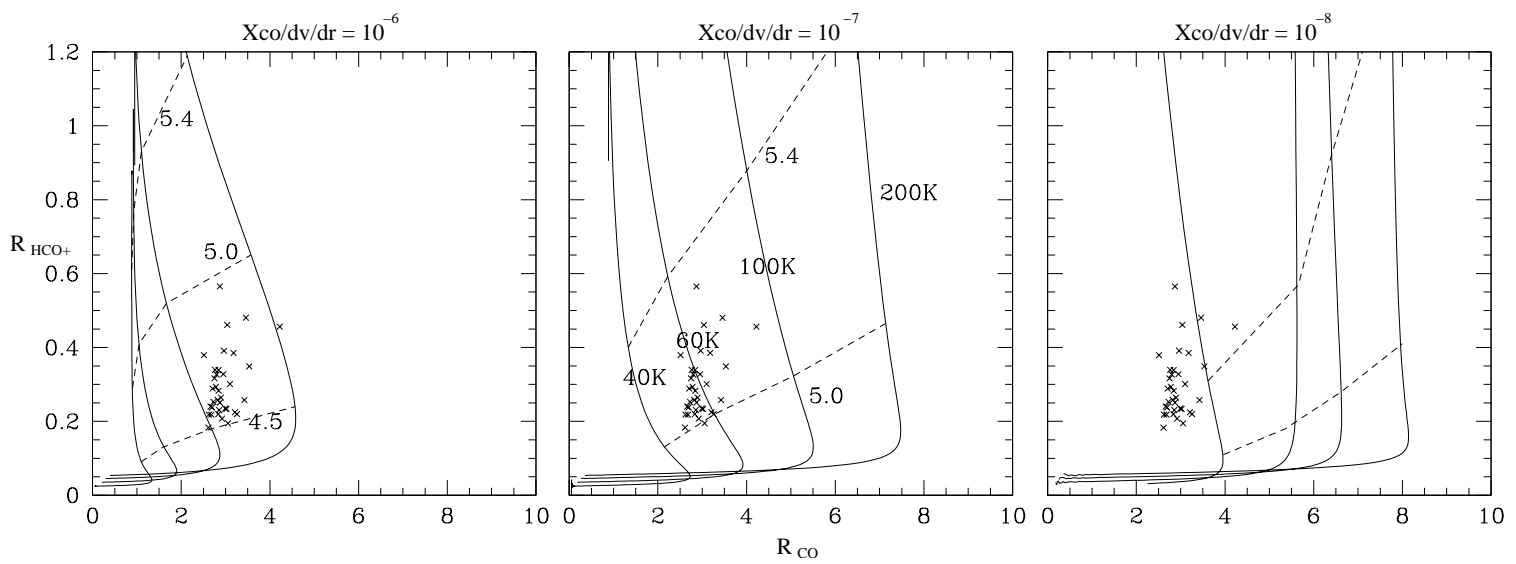

FIG. 10. - The model $\mathrm{R}_{C O}(\mathrm{CO}(3-2) / \mathrm{CO}(1-0))$ vs. $\mathrm{R}_{H C O^{+}}\left(\mathrm{HCO}^{+}(4-3) / \mathrm{HCO}^{+}(1-0)\right)$ shown for different $\mathrm{X}_{C O} /(\mathrm{dv} / \mathrm{dr})$ for comparison. The crosses show the data points from $v=7298 \mathrm{~km} \mathrm{~s}^{-1}$ in Figure9 The solid line shows the temperature contours of 40, 60, 100, and $200 \mathrm{~K}$, and the dashed line shows density contours of $10^{5.0}$ and $10^{5.4} \mathrm{~cm}^{-3}$ (and $10^{4.5} \mathrm{~cm}^{-3}$ for $\left.\mathrm{X}_{C O} /(\mathrm{dv} / \mathrm{dr})=10^{-6}\left(\mathrm{~km} \mathrm{~s}^{-1} \mathrm{pc}-1\right){ }^{-1}\right)$. Using $X_{C O} /(\mathrm{dv} / \mathrm{dr})=10^{-6}\left(\mathrm{~km} \mathrm{~s}^{-1} \mathrm{pc}^{-1}\right)^{-1}$ will shift the temperature (vertical) contours leftward, yielding most of the molecular gas with temperatures in excess of $200 \mathrm{~K}$. Using $X_{C O} /(\mathrm{dv} / \mathrm{dr})=10^{-8}\left(\mathrm{~km} \mathrm{~s}^{-1} \mathrm{pc}^{-1}\right)^{-1}$ will shift the temperature contours rightward, yielding a wide range of temperatures but mostly $\mathrm{T}<60 \mathrm{~K}$. 


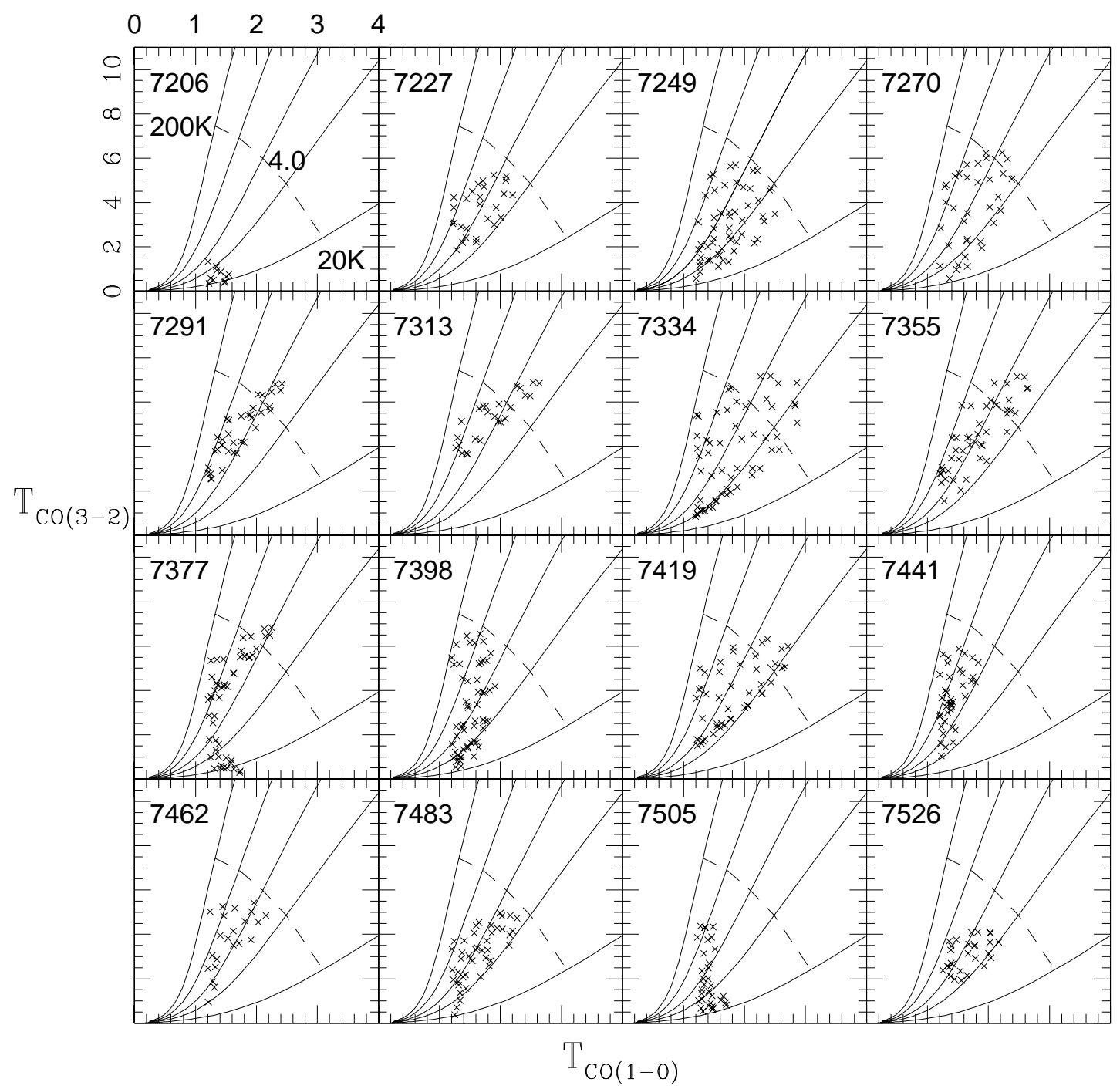

FIG. 11. - The pixel-to-pixel correlation between $\mathrm{T}_{\mathrm{CO}(3-2)}$ and $\mathrm{T}_{\mathrm{CO}(1-0)}$ (in Kelvin) are shown in crosses, where a beam filling factor of unity is assumed. The solid lines show the $\mathrm{T}_{K}=20,40,60,100,200 \mathrm{~K}$, and the dashed line shows $n_{\mathrm{H}_{2}}=10^{4.0} \mathrm{~cm}^{-3}$ contour. 


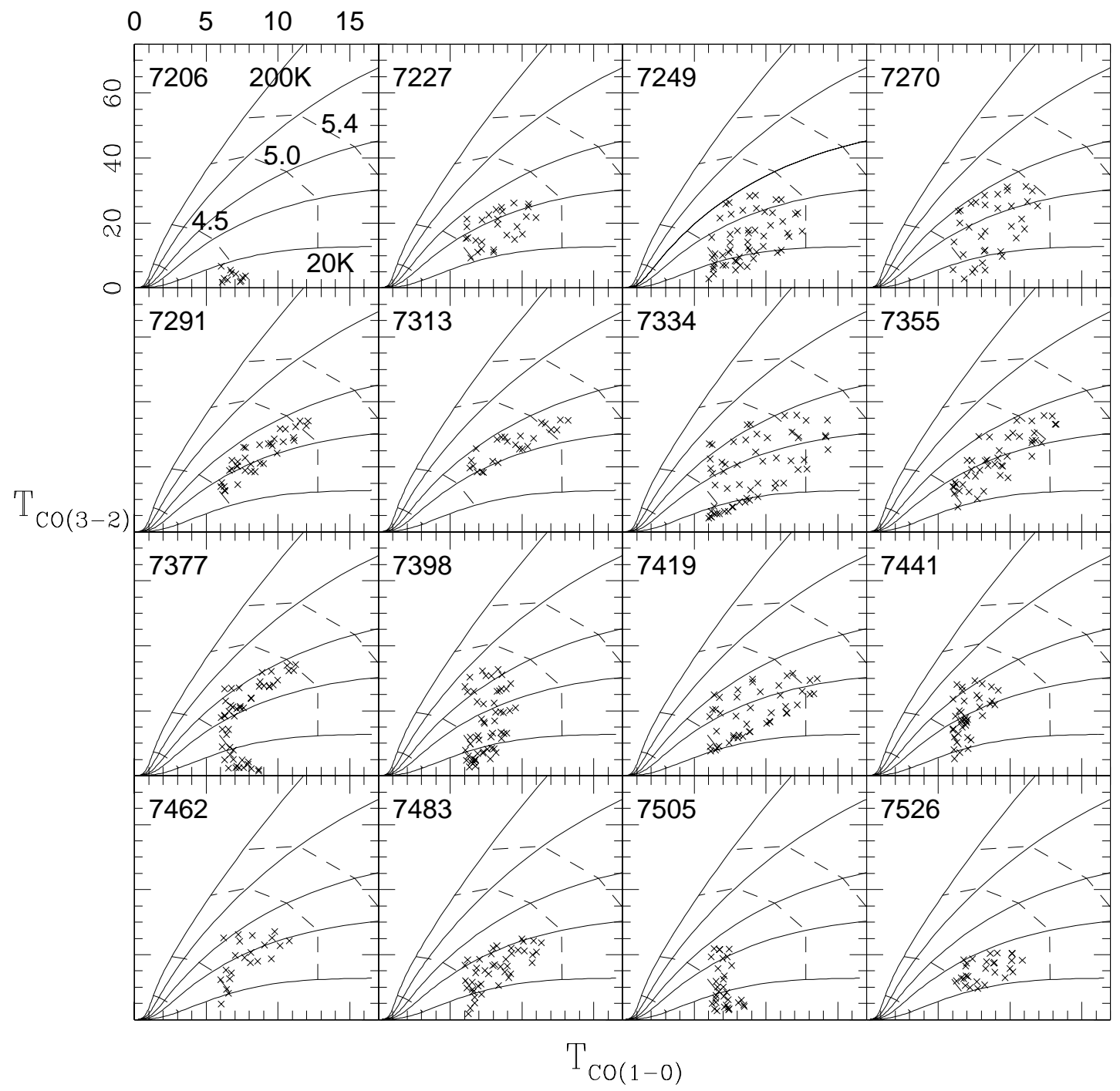

Fig. 12. - Similar to Figure 11 but a beam filling factor of 0.2 is assumed. 


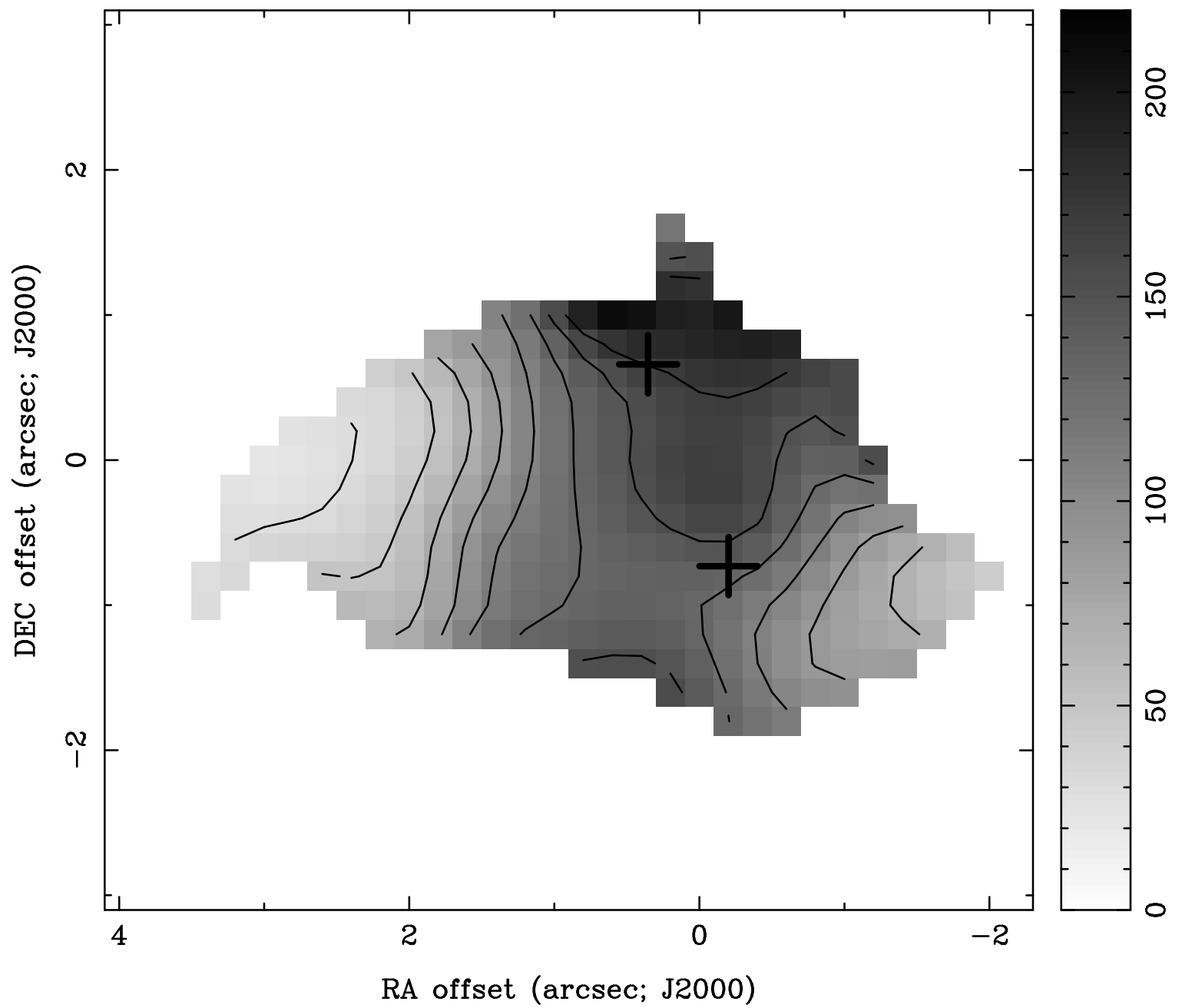

FIG. 13. - The gas-to-dust ratio map where both measurements are above 3 sigma. The contours are 30,50,70,90,110,130,150,170. The crosses are the same as in Figure 4 


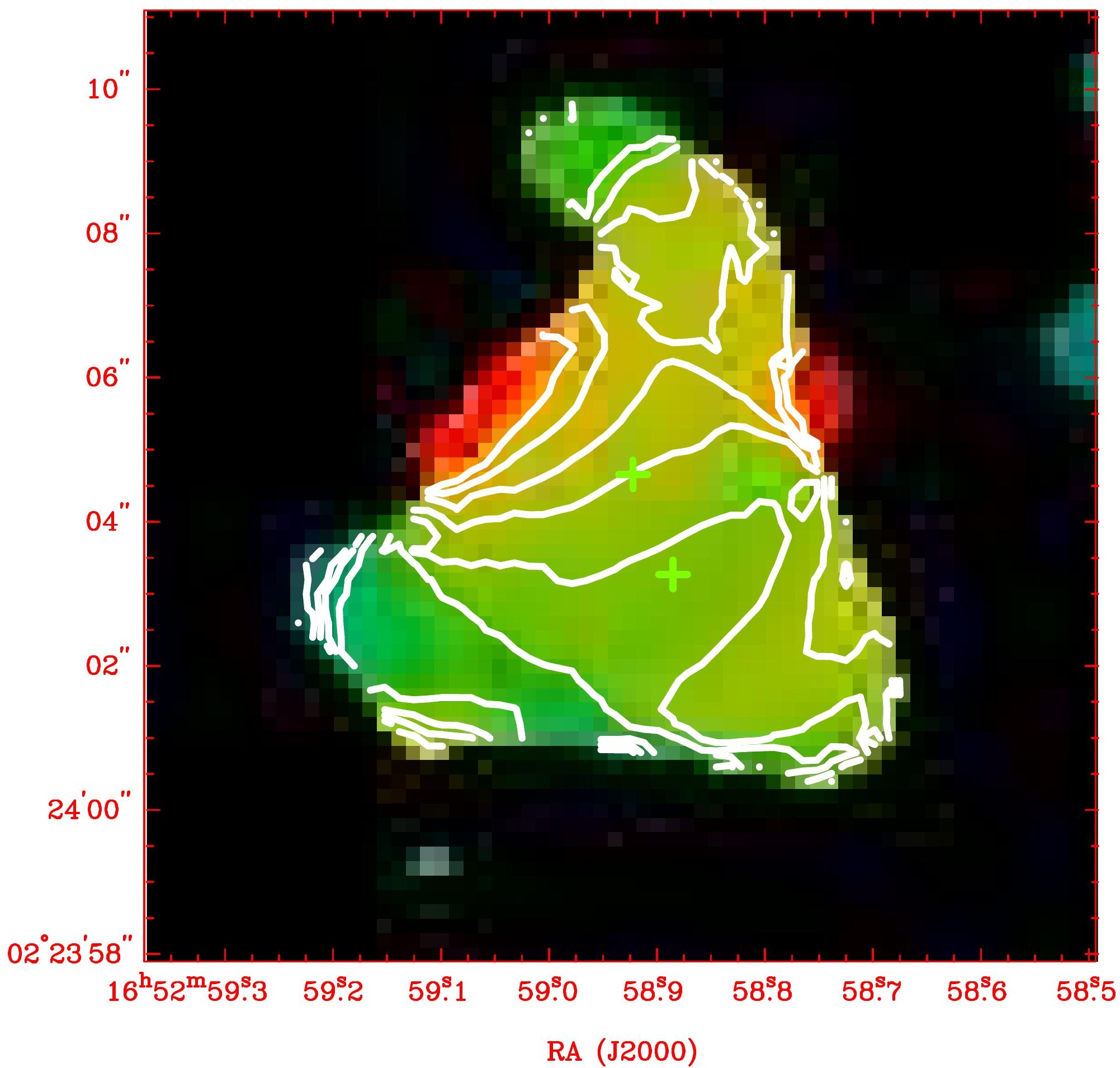




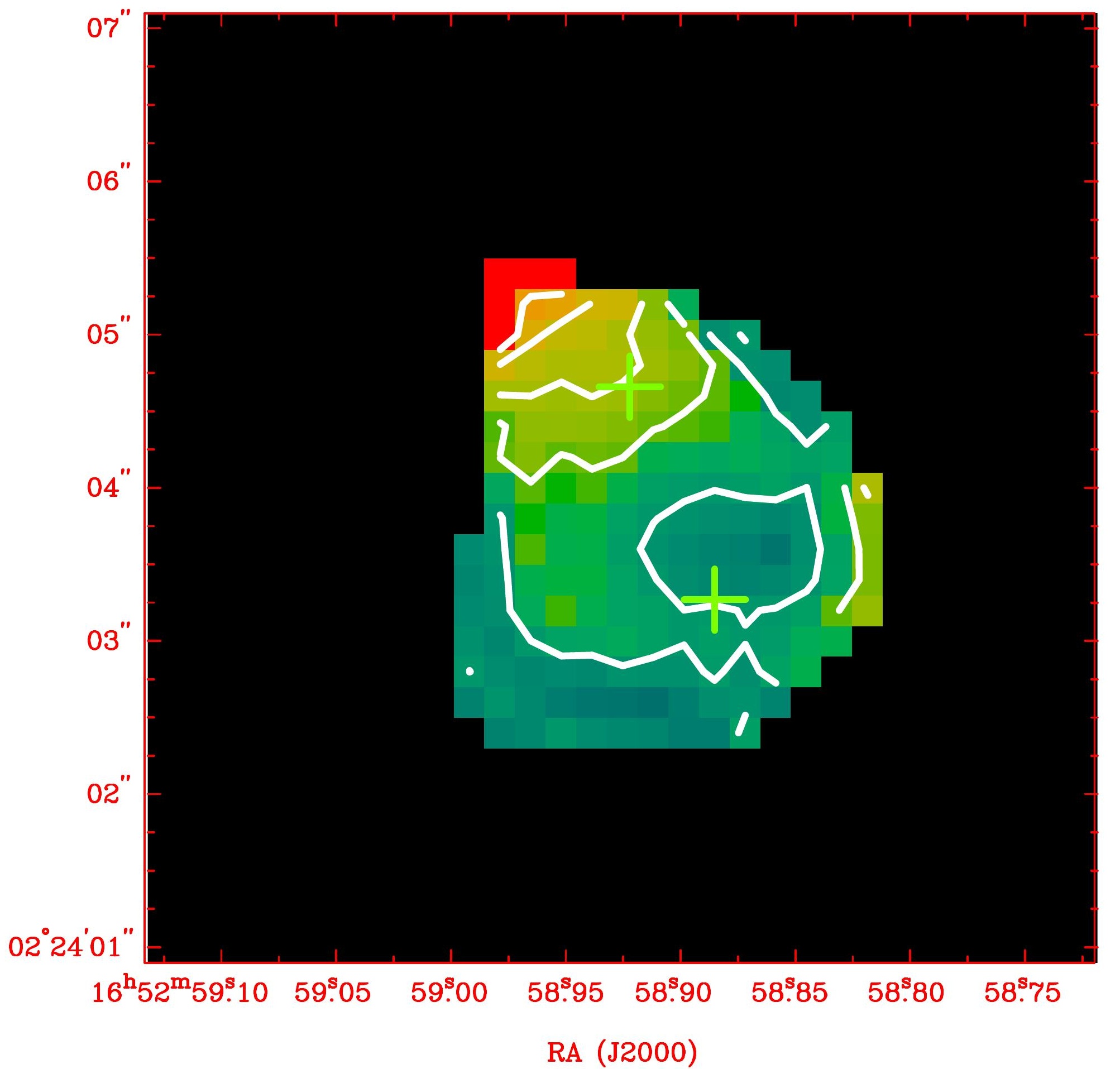




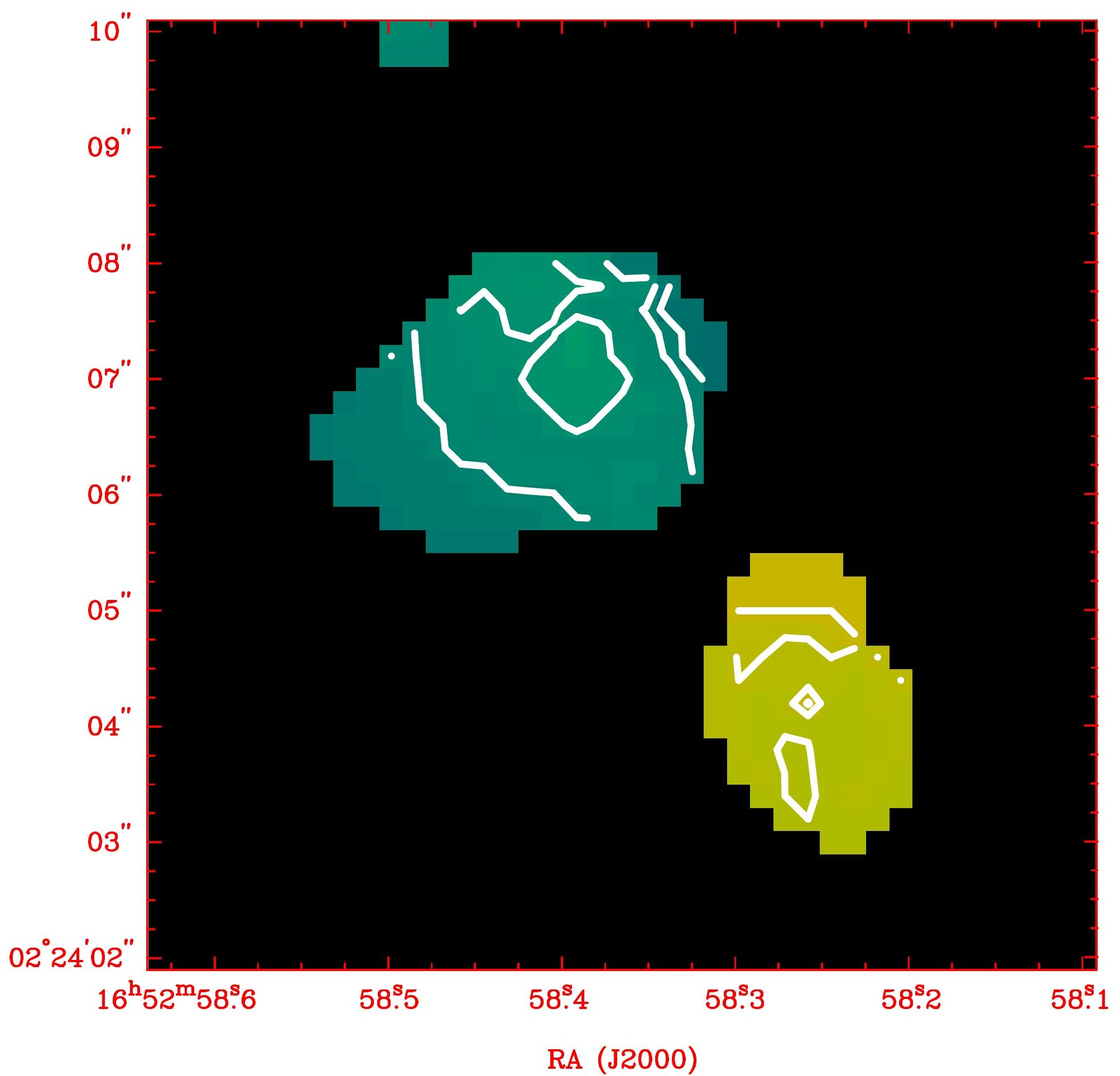

